\title{
Fitosociología y sintaxonomía de los manglares y saladares de las lagunas costeras de los cayos Coco y Sabinal, Cuba
}

\author{
Eddy Martínez-Quesada \\ Centro de Investigaciones de Medio Ambiente de Camagüey (CIMAC) \\ Cisneros No. 105 (altos) /Pobre y Ángel, Camagüey 1. C.P. 70100, Cuba
}

\section{Correspondencia}

E. Martínez-Quesada

e-mail: eddy@cimac.cu

Recibido: 28 marzo 2017

Aceptado: 27 octubre 2017

Publicado on-line: diciembre 2017

\begin{abstract}
Resumen
Se estudió la fitosociología y sintaxonomía de los manglares y saladares asociados a las lagunas costeras de los cayos Coco y Sabinal, en el archipiélago Sabana-Camagüey, al norte de Cuba. Se encontraron 11 asociaciones vegetales, nueve de la clase Rizophoretea mangle y dos de la clase BatidoSarcocornietea. Se describe por primera vez una asociación, que constituye un manglar. En las comunidades estudiadas predominan los nanofanerófitos de hojas micrófilas, debido a la interacción con factores limitantes, como el sustrato, la salinidad y la disponibilidad de agua dulce. La flora sinantrópica acompañante se compone en su mayoría por apófitos, principalmente extrapófitos, como Batis maritima, y carece de antropófitos. La zonación de las comunidades no sigue un patrón fijo. Se presenta un esquema sintaxómico con las clases, órdenes, alianzas y asociaciones estudiadas.
\end{abstract}

Palabras clave: Archipiélago Sabana-Camagüey, fitosociología, sintaxonomía, manglares, saladares.

\begin{abstract}
Phytosociology and syntaxonomy of mangroves and halophilous plant communities of coastal lagoons of Coco and Sabinal cays, Cuba.

The phytosociology and syntaxonomy of mangroves and halophilous plant communities associated to coastal lagoons of Coco and Sabinal cays in the Sabana-Camagüey archipelago, at north of Cuba, were studied. Eleven plant associations were found, nine of them belong the Rizophoretea mangle class and two from the Batido-Sarcocornietea class. One association from mangrove is described for the first time. The nanophanerophytes which present microphyllous leaves are predominant, due to an extreme limitative ecological factors interaction, like substrate, salinity and the availability of fresh water. The synanthropic companion flora is mainly composed by apophytes, chiefly the extrapophytes, such as Batis maritima, and lack anthropophytes. Communities do not show a consistent pattern among zones. A syntaxonomical scheme with classes, orders, alliances and associations studied is included.
\end{abstract}

Key words: Sabana-Camagüey archipelago, phytosociology, syntaxonomy, mangroves, salt marshes.

\section{Introducción}

En Cuba los manglares ocupan aproximadamente el $5 \%$ de la superficie del país y representan el $26 \%$ de su superficie boscosa total estando presentes en las costas biogénicas, acumulativas, cenagosas y con esteros (Menéndez Carrera \& Priego Santander, 1994). En el 
Archipiélago Sabana-Camagüey, los manglares constituyen la formación vegetal mejor representada debido a las condiciones de inundaciones, tanto permanentes como temporales, y a las mareas de ese territorio. Se hallan en las costas bajas resguardadas y los bordes de las lagunas costeras, detrás de las dunas (Menéndez Carrera \& Guzmán Menéndez, 2005, 2007).

La vegetación halófila o saladares, conformada principalmente por comunidades herbáceas, se presenta en lugares donde existe una alta concentración salina influenciada por las inundaciones estacionales. Con una menor extensión que los manglares, se desarrolla en los bordes de las lagunas costeras, o en el interior de ellas durante el periodo poco lluvioso.

El grupo insular de los Jardines del Rey o Sabana-Camagüey se extiende entre la península de Hicacos y la bahía de Nuevitas, a través de 465 $\mathrm{km}$ al norte de la isla Cuba, siendo el de mayor número de cayos e islas. Se subdivide en cinco subgrupos de oeste a este, el último de ellos comprende a los cayos Coco, Romano, Guajaba y Sabinal. Dada la extensión de los mismos y la existencia de un manto freático se han considerado como verdaderas islas (Hernández Santana \& Díaz Díaz, 1989). En estos cayos existen lagunas litorales que tienen intercambio con el mar y los manglares constituyen la principal formación vegetal asociada a ellas. En cayo Coco dichas lagunas representan uno de los ecosistemas que más afectaciones han sufrido por el desarrollo de la infraestructura hotelera (Batista et al., 2006).

Los estudios sintaxonómicos en los manglares y comunidades halófilas realizados en las zonas costeras y cayos adyacentes cubanos han sido pocos y limitados a determinadas localidades del occidente y oriente del país (Borhidi 1991, 1996; Reyes Domínguez \& Acosta Castillo, 2003; Reyes Domínguez, 2006), mientras que en su parte central solo se conoce el trabajo de MartínezQuesada (2014), donde se describen asociaciones nuevas de las clases Rhizophoretea mangle y Batido-Sarcocornietea, en algunos cayos del archipiélago Jardines de la Reina, al sur de la provincia Camagüey. Por ello el conocimiento de las fitocenosis de manglar y de las comunidades halófilas es todavía insuficiente en un territorio que tiene amplias potencialidades para el desarrollo turístico y por lo tanto un incremento de la acción antrópica sobre esos ecosistemas. En la bibliografía consultada no se observan estudios de este tipo en la región central, donde se ubica el mencionado archipiélago, por lo que el objetivo del presente trabajo es estudiar y describir los sintaxones asociados a los manglares

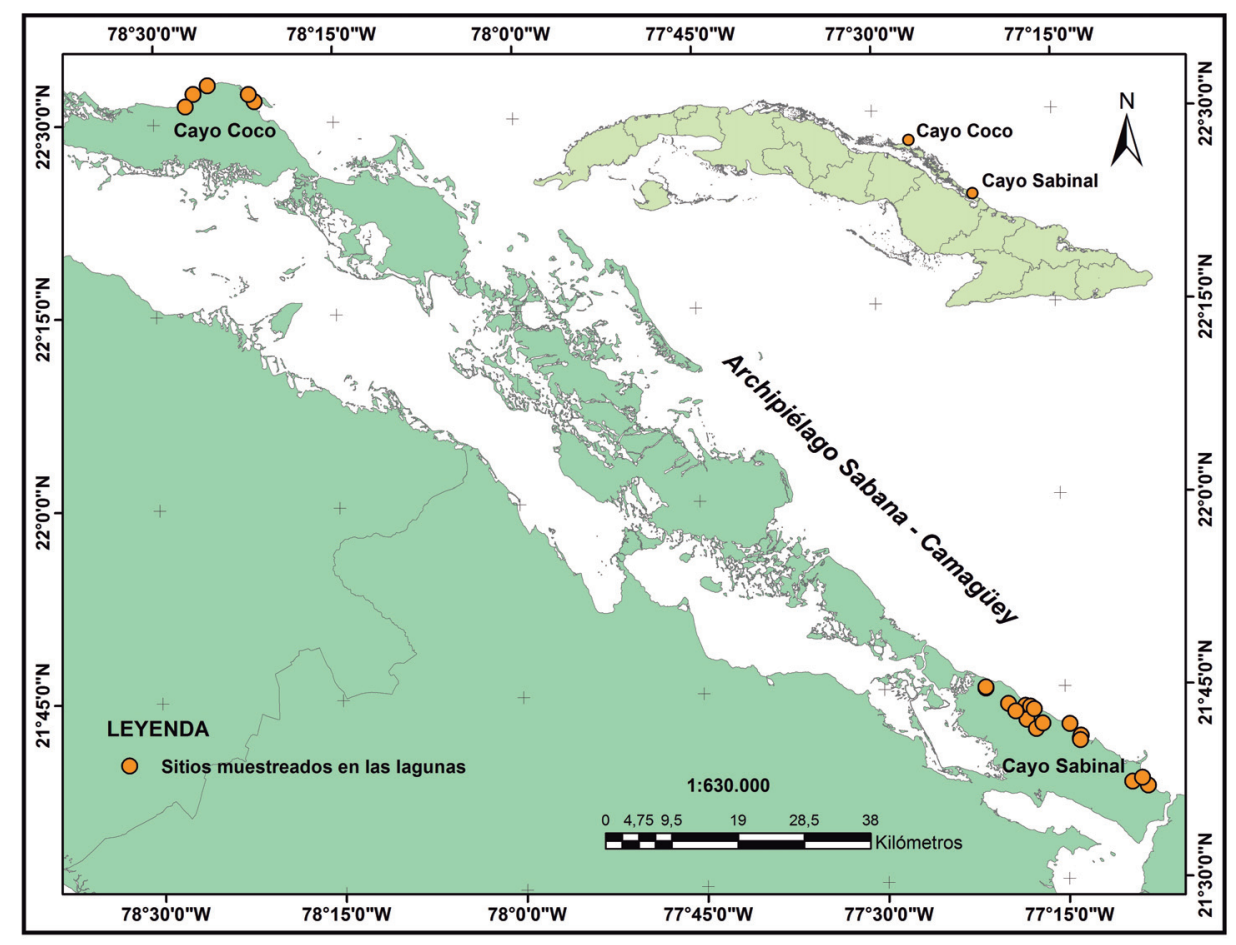

Figura 1. Ubicación de los cayos Coco y Sabinal en el archipiélago Sabana-Camagüey, Cuba (los puntos son los lugares donde se levantaron los inventarios). Figure 1. Location of Coco and Sabinal cays in the Sabana-Camagüey archipelago, Cuba (points are localities where plots where carried out). 
y comunidades halófilas de las lagunas costeras de los cayos Coco y Sabinal.

\section{Material y métodos}

\section{El territorio estudiado}

Cayo Coco ocupa un área de $370 \mathrm{~km}^{2}$. Presenta un relieve llano, con alturas promedio de 2 a $5 \mathrm{~m}$, sobresaliendo dunas de hasta $12 \mathrm{~m}$, en la zona conocida como Alto del Puerto, considerada una de las más altas del país. Cayo Sabinal, por su parte, tiene un área de $335 \mathrm{~km}^{2}$ y posee un relieve llano, con pequeñas elevaciones al sur del cayo que alcanzan hasta $28 \mathrm{~m}$ (Valdés Montero, 2000). En la figura 1 se puede apreciar la ubicación de ambos, como parte del archipiélago SabanaCamagüey.

Fitogeográficamente el territorio donde se ubican estos cayos pertenece a la subprovincia Cuba Central, Sector Cuba Centro-Oriental, Subsector Guaimaricum, distrito Gibarense (Borhidi, 1991).

Toda el área costera está compuesta por complejos litológicos carbonatados-neógenocuaternarios, fuertemente afectados por una densa red de morfoalineamientos y numerosas formas cársticas superficiales que evidencian un intenso proceso de carstificación. El relieve terrestre que predomina es llano (Hernández Santana \& Díaz Díaz, 1989). Las llanuras son de tipo abrasivoacumulativas, planas y parcialmente cenagosas (Portela Peraza et al., 1989) y se presentan pendientes menores de $0,5^{\circ}$ (Magaz García, 1989). El suelo es húmico calcimórfico, del tipo rendzina negra típico (Marrero Rodríguez et al., 1989). La insolación anual es elevada, con 2900 horas de luz o más (Campos Mazorra \& Vigón del Busto, 1989). La temperatura media anual del aire es $24-26^{\circ} \mathrm{C}$ (Lapinel Pedroso, 1989) y la media anual de precipitaciones de 1200-1400 mm, con la media en el periodo lluvioso de $800-1000 \mathrm{~mm}$, y en el periodo seco de 200-400 mm (Izquierdo Ramos, 1989). La humedad relativa media anual a las 7 horas es $90-95 \%$ y las 13 horas, $70-75 \%$ (Lecha, 1989). La evaporación media anual es elevada, con 2000-2200 mm (Crespo González, 1989).

Desde el punto de vista paisajístico, y de acuerdo con Priego Santander et al. (2004), en la zona que ocupan las lagunas se presenta una llanura marino-biógena muy baja $(\leq 1 \mathrm{~m})$ y plana, formada por calizas, depósitos marinos y terrígenos carbonatados, con bosque siempreverde y comunidades herbáceas sobre suelos hidromórficos, que a su vez puede presentar dos unidades: una, de superficie acumulativa muy baja $(\leq 1 \mathrm{~m})$, estacional y por partes permanentemente inundada, con manglar de Rhizophora mangle, manglar mixto y de Conocarpus erectus, comunidades halófilas $y$, por partes, saladares sobre suelos de gley solonchakado, marga costera solonchakada, salinizada y muy salinizada, y solonchak gleyzado; y la otra de lagunas cársticas y de acumulación diferencial, poco profundas $(<2 \mathrm{~m})$, con sedimentos fangosos y areno-fangosos rodeadas de manglares y comunidades halófilas.

\section{Procesamiento de inventarios, nomenclatura y colecciones}

Los inventarios de vegetación se levantaron según el método fitosociológico de Braun Blanquet (1979) en los meses abril y septiembre, desde el 2014 hasta el 2016, en los cayos Coco y Sabinal. Para su confección, se efectuaron recorridos en sitios puntuales por el norte y el sur de las lagunas costeras previamente identificadas. En todos los casos se tomaron las coordenadas geográficas con un GPS. Se tuvieron en cuenta las características y variaciones florísticas de la vegetación, como la estructura vertical y composición florística, así como también los tipos de suelos y la presencia o no de agua según la época del año. El área mínima se calculó mediante el establecimiento de parcelas cuadradas, con un área de $2 \times 2$ metros como mínimo, en la cual se anotaron todas las especies presentes y se observó si había un incremento significativo de las mismas, en cuyo caso se duplicó el tamaño de dicha parcela para valorar si se incrementaban las especies. De lo contrario el área mínima quedaba establecida como la menor área con la mayor cantidad de especies. Así, fue de $16 \mathrm{~m}^{2}$ para los manglares y $4 \mathrm{~m}^{2}$ para la vegetación halófila. En cada inventario se estimó la abundancia-dominancia de las especies por estratos y sinusias, según los valores de la escala combinada de Braun Blanquet (1979). Se levantaron un total de 108 inventarios, los que se compararon con aquellos referidos por Borhidi (1991, 1996), Peinado et al. (1995), Reyes Domínguez \& Acosta Cantillo (2003), Reyes Domínguez (2006) y Martínez-Quesada (2014). Se consideraron principalmente dos estratos, el arbóreo (E3), generalmente para las especies mayores de $5 \mathrm{~m}$ y el herbáceo (E1), para las que alcanzan hasta $2 \mathrm{~m}$ de altura, según las descripciones y nomenclatura de Samek (1973). Sin embargo, en aquellos casos donde las condiciones del edátopo son extremas, no permitiendo el desarrollo de los árboles, el E3 bien delimitado se consideró por debajo de los 5 $\mathrm{m}$ de altura. El E2 generalmente no se desarrolla en los manglares; sin embargo, se tuvo en cuenta por debajo de los $2 \mathrm{~m}$ de altura, cuando estuvo definido. 


\begin{tabular}{|c|c|c|c|c|}
\hline Taxon & Comunidades & Tipo sinantrópico & Tipo biológico & Tipo de hoja \\
\hline Ambrosia hispida & 6 & - & caméfito & notófila \\
\hline Avicennia germinans & $2,3,4,5,9,10$ & - & microfanerófito & micrófila \\
\hline Batis maritima & $2,3,4,5,6,9,10$ & extrapófito & nanofanerófito & leptófila \\
\hline Borrichia arborescens & 2,5 & intrapófito recurrente & nanofanerófito & nanófila \\
\hline Broughtonia lindenii & 6 & - & epífita arborícola & - \\
\hline Bucida molinetii & 9 & - & microfanerófito & micrófila \\
\hline Casasia clusiifolia & 8 & - & microfanerófito & notófila \\
\hline Cienfuegosia yucatanensis & 6 & - & nanofanerófito & leptófila \\
\hline Conocarpus erectus & $2,4,5,6,7,8,9,11$ & - & macrofanerófito & micrófila \\
\hline Cynanchum caribaeum & 6 & - & trepadora & leptófila \\
\hline Distichlis spicata & 9 & - & hemicriptófito & - \\
\hline Ernodea littoralis & 8 & - & nanofanerófito & nanófila \\
\hline Erithalis fruticosa & 8 & intrapófito pionero & nanofanerófito & micrófila \\
\hline Evolvulus convolvuloides & 6 & intrapófito pionero & caméfito & leptófila \\
\hline Fimbristylis cymosa & 6 & parapófito & caméfito & - \\
\hline Fimbristylis ferruginea & 11 & - & caméfito & - \\
\hline Flaveria linearis & 2 & - & nanofanerófito & nanófila \\
\hline Jacquemontia havanensis & 8 & intrapófito pionero & nanofanerófito & leptófila \\
\hline Jacquinia keyensis & 9 & - & microfanerófito & micrófila \\
\hline Iva imbricata & 6 & - & caméfito & micrófila \\
\hline Lantana involucrata & 8 & intrapófito recurrente & nanofanerófito & nanófila \\
\hline Laguncularia racemosa & $2,5,7,9$ & - & macrofanerófito & micrófila \\
\hline Pilosocereus royenii & 9 & - & suculenta & - \\
\hline Pithecellobium keyense & 6 & - & microfanerófito & micrófila \\
\hline Pithecellobium mucronatum & 9 & - & microfanerófito & micrófila \\
\hline Rachicallis americana & 6 & - & nanofanerófito & leptófila \\
\hline Rhizophora mangle & $1,2,4,5$ & - & macrofanerófito & micrófila \\
\hline Sarcocornia perennis & $2,3,10,11,5,6,9$ & - & nanofanerófito & - \\
\hline Selenicereus grandiflorus & 6 & extrapófito & suculenta & - \\
\hline Senna chapmanii (Isely) & 6 & - & microfanerófito & micrófila \\
\hline Sida ciliaris var. ciliaris & 6 & - & caméfito & leptófila \\
\hline Sideroxylon horridum & 9 & - & microfanerófito & micrófila \\
\hline Sesuvium maritimum & 6 & - & caméfito & leptófila \\
\hline Sesuvium portulacastrum & $2,5,10,6,9$ & - & caméfito & nanófila \\
\hline Sporobolus virginicus & $2,11,6,9$ & - & hemicriptófito & - \\
\hline Suaeda linearis & 11,6 & - & nanofanerófito & nanófila \\
\hline Suriana maritima & $2,4,8$ & - & nanofanerófito & nanófila \\
\hline Tabebuia trachycarpa & 6 & intrapófito recurrente & nanofanerófito & nanófila \\
\hline Tillandsia paucifolia & 9 & - & epífita arborícola & - \\
\hline
\end{tabular}

Tabla 1. Relación de especies que integran las asociaciones estudiadas, tipos sinantrópicos, biológicos y de hojas. Table 1. Species of studied associations, sinantropic types, life forms and leaf types. 1. Rhizophoretum mangle, 2. Batido-Avicennietum germinantis, 3. Avicennietum germinantis, 4. Avicennio germinantis-Rhizophoretum mangle, 5. Lagunculario racemosae-Rhizophoretum mangle, 6. Batido-Conocarpodetum erecti, 7. Lagunculario racemosae-Conocarpodetum erecti, 8. Conocarpodetum erecti, 9. Sporobolo virginici-Conocarpodetum erecti, 10. Batidetum maritimae, 11. Sarcocornietum perennis. 
Tabla 2

\begin{tabular}{|c|c|c|c|c|c|}
\hline \multicolumn{6}{|c|}{$\begin{array}{c}\text { Rhizophoretum mangle Cuatrecasas } 1958 \\
\text { (Rhizophoretea mangle, Rhizophoretalia, Rhizophorion occidentalis) }\end{array}$} \\
\hline Inventario $\mathrm{n}^{\circ}$ & 104 & 105 & 106 & 107 & 108 \\
\hline Estrato arbóreo E3 (\%) & 90 & 90 & 95 & 90 & 90 \\
\hline $\mathrm{N}^{\circ}$ especies & 1 & 1 & 1 & 1 & 1 \\
\hline \multicolumn{6}{|c|}{ Características de asociación y unidades superiores } \\
\hline Rhizophora mangle (E3) & 5.5 & 5.5 & 5.5 & 5.5 & 5.5 \\
\hline
\end{tabular}

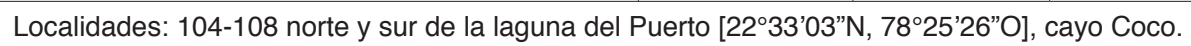

Para la definición de las formaciones vegetales, que conforman las fitocenosis, se utilizó la clasificación de la vegetación de Capote \& Berazaín (1984). Los tipos biológicos son los de Galán de Mera et al. (1999). Por su parte, los tipos de hojas se clasificaron según Borhidi (1976). La categorización de las especies sinantrópicas se realizó según el criterio de Ricardo et al. (1995). La relación entre especies, asociaciones estudiadas, tipo sinantrópico, biológico y tipo de hoja se muestran en la tabla 1.

Para esquematizar la zonación de las comunidades en cada laguna, se realizaron perfiles de vegetación, orientados de norte a sur, mediante el establecimiento de transectos, de acuerdo con Trejo \& Dirzo (2002). Como la extensión de las lagunas y la ubicación de las comunidades es variable, debido a las condiciones ecológicas que se presentan, los transectos no se replicaron hasta 10 veces sino que extendieron hasta los bordes de las lagunas y de esta forma se delimitaron entre 475 m como mínimo y 2000 m como máximo, lo que permite incluir a todas las comunidades. En todos los casos se anotaron las especies presentes hasta un ancho de $2 \mathrm{~m}$ como máximo.

En la descripción de los estratos y sinusias, se establecieron las siguientes categorías de presencia de las especies, de acuerdo con Reyes Domínguez (2005): Constantes (presentes en el $80 \%$ o más de las listas), frecuentes: $60-79 \%$, menos frecuentes: $30-59 \%$, y ocasionales: $15-$ $29 \%$. Las especies con valores menores al $15 \%$ no se nombraron en la descripción. La ordenación de las listas y la separación de los sintaxones se efectuó por métodos fitocenológicos, según Scamoni \& Passarge (1963). Para la categorización y nomenclatura de los rangos principales de las fitocenosis se siguió el Código Internacional de Nomenclatura Fitosociológica (Weber et al., 2000).
Los rangos de clase, orden y alianza a los que pertenecen las asociaciones descritas están de acuerdo con Galán de Mera \& Vicente Orellana (2006).

La identificación de las especies se efectuó directamente en el campo y las dudosas se determinaron posteriormente mediante la utilización de las descripciones morfológicas que aparecen en León (1946) y León \& Alain (1951), así como también mediante la comparación con especímenes de herbarios. Los materiales herborizados que justifican la mención de las especies inventariadas se encuentran depositados en el herbario HACC. Los nombres científicos y las autorías han sido corroborados con el inventario de Greuter \& Rankin Rodríguez (2016) y la base de datos The Plant List (2017).

\section{Resultados y discusión}

\section{Los manglares de las lagunas costeras}

\section{RHIZOPHORETALIA Cuatrecasas 1958}

\section{Rhizophoretum mangle Cuatrecasas 1958}

[Tabla 2, fig. 2A]

Constituye un manglar puro de Rhizophora mangle L. permanentemente inundado sobre sedimentos arenosos. Su areal biogeográfico en Cuba abarca todas las costas por el norte y sur del territorio nacional. En las lagunas estudiadas su distribución es en cayo Coco, por el norte y oeste de laguna Tiburón, norte y sur de laguna Larga, norte y sur de laguna del Puerto, y sur de laguna Las Coloradas; mientras que en cayo Sabinal se halla al norte y sur de laguna Tortuguilla, en todos los casos ubicado en terrenos permanentemente inundados, tanto en el interior como en el canal de 

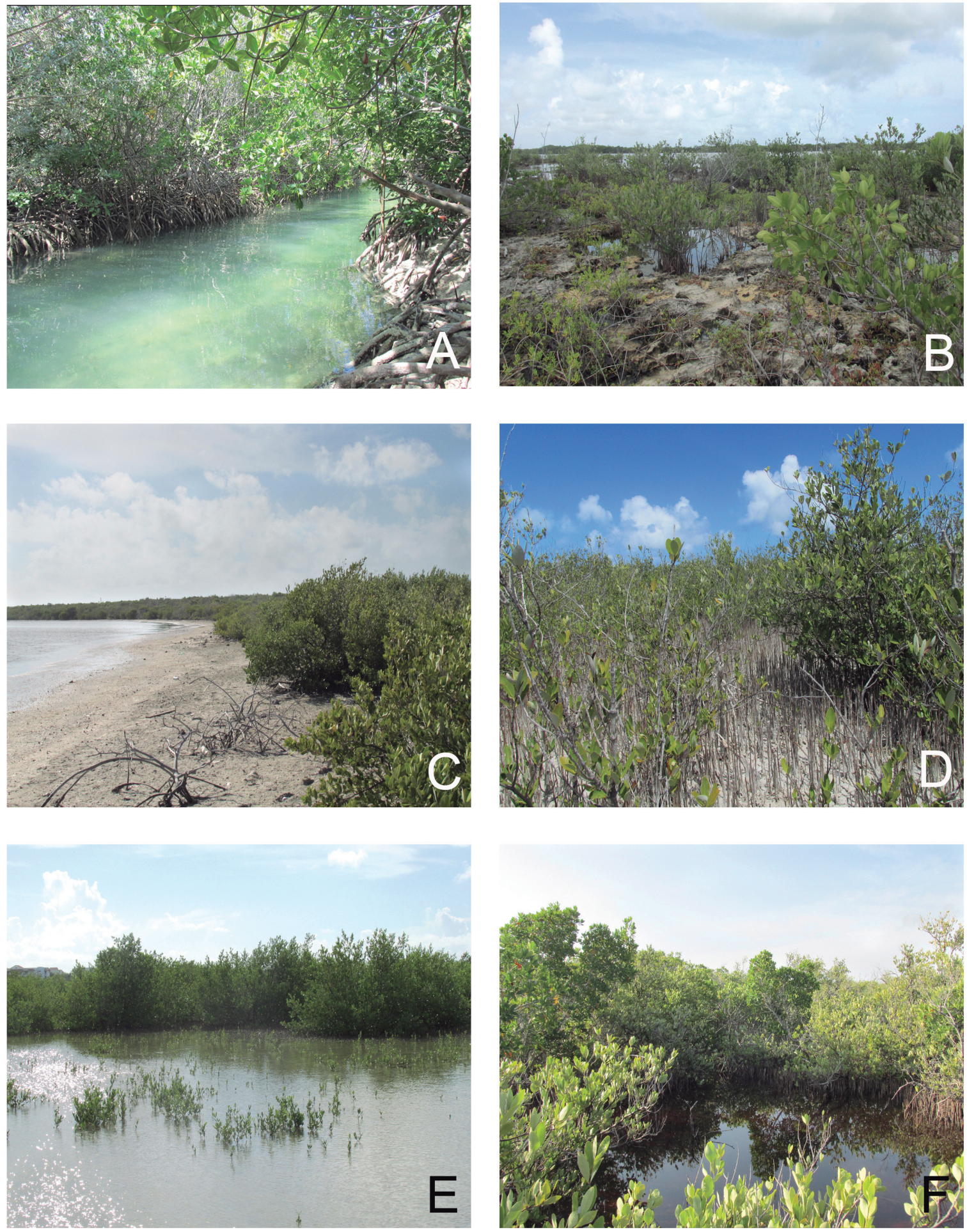

Figura 2. Fitocenosis de manglares en las lagunas costeras. A) Rhizophoretum mangle en el canal de entrada de Laguna Larga, B) Batido-Avicennietum germinantis al sur de la laguna del Puerto, sobre pavimento, C) Oeste de la Laguna Las Coloradas, sobre arena y pavimento, Cayo Coco, D) Avicennietum germinantis en el interior de la Laguna Tiburón durante el período poco lluvioso, E) La misma asociación y localidad en periodo lluvioso, F) Avicennio germinantisRhizophoretum mangle, al norte de la Laguna Tortuguilla, cayo Sabinal. Figure 2. Mangrove phytocoenosis in the coastal lagoons. A) Rhizophoretum mangle at the entrance canal of Larga Lagoon, B) Batido-Avicennietum germinantis, on limestone pavement, at south of Puerto Lagoon, C) West of Las Coloradas lagoon, on sand and limestone pavement, D) Avicennietum germinantis inside Tiburón Lagoon during dry season, E) The same association and locality during rainy season, Coco Cay, F) Avicennio germinantis-Rhizophoretum mangle at North of Tortuguilla Lagoon, Sabinal Cay. 
entrada de dichas lagunas.

El estrato arbóreo cubre entre el 90 y $95 \%$ y tiene una altura de 6 a $9 \mathrm{~m}$. La especie dominante absoluta es Rizhophora mangle, un macrofanerófito de hojas micrófilas, no sinantrópico (tab. 1).

A diferencia del Rhizophoretum mangle mencionado por Reyes Domínguez \& Acosta Cantillo (2003) en nuestro territorio está expuesto directamente al oleaje del mar, por la posición interna que ocupa en las lagunas.

\section{AVICENNIETALIA Cuatrecasas 1958}

\section{Batido-Avicennietum germinantis Borhidi \& Del}

\section{Risco in Borhidi 1991}

[Tabla 3, fig. 2 B y C]

Es un manglar que se desarrolla sobre pavimento carstificado o suelos areno-fangosos, temporalmente inundados. Su areal biogeográfico conocido es la Península de Zapata, parte central de Cuba y delta del río Cauto, en la oriental. En cayo Coco se localiza por el sur de la laguna del Puerto, norte y sur de la laguna Tiburón, y norte y oeste de la laguna Las Coloradas; por su parte en cayo Sabinal, al norte de las lagunas Los
Caimanes y La Salina, así como norte y sur de la laguna Tortuguilla.

El estrato arbóreo presenta una cobertura del 80 a $90 \%$ y una altura de 6 a $10 \mathrm{~m}$. En este la especie constante y muy abundante es Avicennia germinans (L.) L., la frecuente es Conocarpus erectus L. y la menos frecuente, Laguncularia racemosa (L.) C.F. Gaertn.

El estrato arbustivo, muy bajo, se observa cuando se presenta sobre pavimento carstificado liso, al sur de la laguna del Puerto o sustrato arenoso con partes pavimentadas, al oeste de Las Coloradas. En este la cobertura es del 25 a 90\% y la altura oscila entre 1,4 a $2 \mathrm{~m}$. La constante y muy abundante es la propia Avicennia germinans, que al igual que en los otros mangles se restringe a las oquedades del pavimento carstificado. El estrato herbáceo posee una cobertura del 50 a $95 \%$ y una altura de 20 a $50 \mathrm{~cm}$. La especie constante, y por lo general abundante, es Batis maritima L., pero también Avicennia germinans tiene en este estrato una buena cantidad de individuos jóvenes, la frecuente es Sarcocornia perennis (Mill.) A.J. Scott, y la ocasional, Sporobolus virginicus (L.) Kunth.

Tabla 3

\begin{tabular}{|c|c|c|c|c|c|c|c|c|c|c|c|c|}
\hline \multicolumn{13}{|c|}{$\begin{array}{l}\text { Batido-Avicennietum germinantis Borhidi y Del Risco in Borhidi } 1991 \\
\text { (Rhizophoretea mangle, Avicennietalia, Avicennion occidentalis) }\end{array}$} \\
\hline Inventario $\mathrm{n}^{\circ}$ & 31 & 32 & 33 & 1 & 2 & 4 & 68 & 69 & 70 & 72 & 26 & 25 \\
\hline E3 Cobertura (\%) & 85 & 90 & 80 & 90 & 85 & 90 & - & - & - & - & - & - \\
\hline E2 Cobertura (\%) & - & - & - & - & - & - & 50 & 45 & 25 & 50 & 90 & 85 \\
\hline E1 Cobertura (\%) & 90 & 95 & 95 & 95 & 60 & 60 & 75 & 75 & 90 & 75 & 90 & 75 \\
\hline $\mathrm{N}^{\circ}$ especies & 5 & 4 & 5 & 4 & 7 & 5 & 5 & 4 & 5 & 11 & 5 & 5 \\
\hline \multicolumn{13}{|c|}{ Características de asociación y unidades superiores } \\
\hline $\begin{array}{l}\text { Avicennia germinans } \\
(\mathrm{E} 3,2,1)\end{array}$ & 5.1 & 4.1 & 2.1 & 4.1 & 4.1 & 5.1 & 4.1 & 4.1 & 3.1 & 4.1 & 5.5 & 5.5 \\
\hline Batis maritima (E1) & 5.5 & 5.5 & 5.5 & 5.5 & 3.5 & 3.5 & 4.2 & 3.2 & 4.2 & 3.5 & 2.1 & 5.5 \\
\hline Conocarpus erectus (E3) & 2.1 & 3.1 & 4.1 & 3.1 & 2.1 & 3.1 & . & $\sigma^{\prime}$ & . & 3.1 & r.1 & . \\
\hline Laguncularia racemosa & r.1 & r.1 & . & 1.1 & +.1 & . & . & & r.1 & 1.1 & $\cdot$ & . \\
\hline $\begin{array}{l}\text { Sesuvium portulacastrum } \\
\text { (E1) }\end{array}$ & & . & 3.2 & . & +.1 & . & . & & . & r.2 & & r.1 \\
\hline Rhizophora mangle (E3 ) & r.1 & . & . & . & . & . & r. 1 & & . & 2.1 & & . \\
\hline Sarcocornia perennis (E1) & . & . & . & . & +.1 & +.1 & +.2 & 3.2 & 2.2 & +.1 & 1.1 & 3.1 \\
\hline \multicolumn{13}{|l|}{ Compañeras } \\
\hline Sporobolus virginicus & . & . & +.2 & . & . & . & . & . & . & r.2 & 2.2 & 4.3 \\
\hline Sesuvium maritimum & & . & . & . & . & . & 3.2 & 3.2 & 3.2 & . & & . \\
\hline Salicornia bigelovi & . & . & . & . & +.1 & +.1 & . & & . & +.1 & . & . \\
\hline
\end{tabular}

Otras compañeras: En 2: Borrichia arborescens +.1, En 72: Suriana maritima +.1, Flaveria linearis +.1.

Localidades: $1-4$ norte de la laguna Tiburón [22 $32^{\prime} 24^{\prime \prime} \mathrm{N}, 78^{\circ} 26^{\prime} 38^{\prime \prime} \mathrm{O}$ ], 25-26 oeste de la laguna Las Coloradas

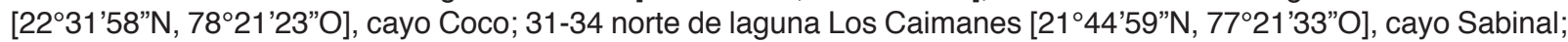
$68-72$ sur de la de laguna del Puerto [ $22^{\circ} 33^{\prime} 03^{\prime \prime} \mathrm{N}, 78^{\circ} 25^{\prime} 26^{\prime \prime} \mathrm{O}$ ], cayo Coco. 
Tabla 4

Avicennietum germinantis Reyes \& Acosta 2003

(Rhizophoretea mangle, Avicennietalia, Avicennion occidentalis)

\begin{tabular}{|l|l|l|l|l|l|}
\hline Inventario $\mathrm{n}^{\circ}$ & 9 & 10 & 11 & 12 & 13 \\
\hline Estrato arbóreo E3 (\%) & 90 & 75 & 85 & 90 & 90 \\
\hline Estrato herbáceo E1 (\%) & disp. & disp. & disp. & disp. & disp. \\
\hline $\mathrm{N}^{\circ}$ especies & 3 & 2 & 1 & 1 & 1 \\
\hline
\end{tabular}

Características de asociación y unidades superiores

\begin{tabular}{|l|l|l|l|l|l|}
\hline $\begin{array}{l}\text { Avicennia germinans } \\
\text { (E3) }\end{array}$ & 5.5 & 5.5 & 5.5 & 4.5 & 5.5 \\
\hline Compañeras \\
\hline Batis maritima (E1) & +.2 &. &. &. &. \\
\hline
\end{tabular}

Localidades: 9-13 interior de la laguna Tiburón [22'32'24”N, 78²6’38'O], cayo Coco. disp. = disperso

La asociación es pobre en especies, posee 5,2 como promedio y ninguna es endemismo. Se compone mayormente de nanofanerófitos, con predominio de las hojas micrófilas y nanófilas. Dos especies son sinantrópicas: Batis maritima, un extrapófito, y Borrichia arborescens (L.) DC., un intrapófito recurrente, en un $15,3 \%$ en la cenosis (tab. 1).

El desarrollo de esta fitocenosis sobre el pavimento carstificado, conjuntamente con la disponibilidad de agua dulce y el aumento de la salinidad, tiene implicaciones sobre su fisionomía, ya que las especies de mangles no llegan a ser verdaderos árboles; mientras que en zonas con desarrollo del suelo y presencia de humedad sí lo son. Esta puede ser la razón por la cual Reyes Domínguez (2006) presenta a BatidoAvicennietum germinantis como un herbazal, donde Avicennia germinans alcanza una altura hasta $2,5 \mathrm{~m}, \mathrm{y}$ al mismo tiempo se considere un estrato arbustivo, achaparrado, constituido por $A$. germinans. Peinado et al. (1995) refirieron que esta asociación se encuentra en depresiones del terreno donde el agua marina se estanca y luego se seca en la época en que llueve menos, por lo que la concentración salina es elevada en el suelo y debido a esto dicha especie crece hasta 1,5 m. En este estudio se pudo apreciar que cuando $A$. germinans está sobre dicho pavimento la altura del estrato establecido es por lo general de $2 \mathrm{~m}$ y no aumenta significativamente en la época lluviosa, mientras que en el sustrato arenoso, la altura del mismo aumenta significativamente de la época seca a la lluviosa. Esta comunidad al depender de la estacionalidad está influenciada por las precipitaciones, que al ser menores propician un aumento de la salinidad, pero el tipo de sustrato determina la altura del estrato.

\section{Avicennietum germinantis Reyes Domínguez \&} Acosta Cantillo 2003

[Tabla 4, fig. 2 D, E]

Constituye un manglar casi puro de Avicennia germinans, sobre suelo fangoso, temporalmente inundado. Su areal biogeográfico conocido es el delta del rio Cauto, en la parte oriental de Cuba. Se observó en el interior de la laguna Tiburón, por el norte, en cayo Coco.

El estrato arbóreo-arbustivo tiene una cobertura del 75 a $90 \%$ y una altura de 1,70 a 2,5

Tabla 5

\begin{tabular}{|l|l|l|l|l|l|l|l|l|l|}
\hline \multicolumn{7}{|c|}{$\begin{array}{l}\text { Avicennio germinantis-Rhizophoretum mangle Reyes \& Acosta } 2006 \\
\text { (Rhizophoretea mangle, Avicennietalia, Avicennion occidentalis) }\end{array}$} \\
\hline Inventario n & 45 & 46 & 47 & 48 & 49 & 50 & 51 & 52 \\
\hline Estrato arbóreo E3 (\%) & 85 & 85 & 90 & 85 & 90 & 90 & 90 & 90 \\
\hline Estrato herbáceo E1 (\%) & 50 & dis. & dis. & dis. & dis. & dis. & dis. & dis. \\
\hline No especies & 2 & 2 & 4 & 3 & 3 & 4 & 4 & 3 \\
\hline Características de asociación y unidades superiores & 5.5 & 4.5 & +.1 & 3.5 & 5.5 & 5.5 & 2.2 & 4.4 \\
\hline Avicennia germinans (E3,1) &. & +.1 & 4.4 & 2.1 & r.1 & 3.3 & 3.3 & 2.2 \\
\hline Rhizophora mangle (E3) &. &. & r.1 & 2.1 & r.1 & 3.1 & 3.1 & 2.1 \\
\hline Conocarpus erectus & 3.4 &. &. &. &. & 1.2 & +.2 &. \\
\hline Batis maritima (E1) &. &. & r.1 &. &. &. &. \\
\hline Suriana marítima & & & & & &. \\
\hline
\end{tabular}

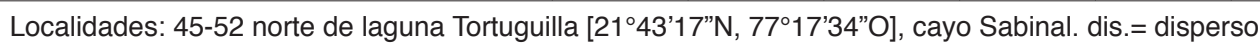


m. La especie dominante absoluta es A.germinans. Por su parte, el herbáceo está disperso. Solo se presentan Batis maritima y Sarcocornia perennis.

Es muy pobre en especies, posee 1,6 como promedio y ninguna es endemismo. Se compone de nanofanerófitos y microfanerófitos y predominan las hojas micrófilas. Posee un extrapófito, Batis maritima, en un $33,3 \%$ en la cenosis (tab. 1).

Esta fitocenosis se ve favorecida durante la época lluviosa, momento en el que supera los 2,5 m de altura, a lo que también contribuye la disminución de los valores de la salinidad. Coincide con lo observado por Reyes Domínguez (2006), en que la pérdida de agua y el aumento de salinidad provocan la forma achaparrada de la misma. Según Mackee (1993) los manglares dominados por A.germinans presentan suelo altamente reductor y un alto contenido de sulfuros. Esas características, unidas a la alta concentración salina durante la época poca lluviosa con periodos de inundación, son la causa de la baja riqueza de especies en esta fitocenosis.

\section{Avicennio germinantis-Rhizophoretum mangle}

Reyes Domínguez \& Acosta Cantillo in Reyes Domínguez 2006

[Tabla 5, fig. 2F]

Es un manglar sobre un suelo areno-fangoso, temporalmente inundado. Su areal biogeográfico conocido en Cuba es el delta del río Cauto, en la parte oriental de Cuba. En este estudio se encontró al norte de la laguna Tortuguilla, en cayo Sabinal.
El estrato arbóreo presenta una cobertura del 85 a $90 \%$ y una altura de 2 a $7 \mathrm{~m}$. La especie constante y muy abundante es Avicennia germinans. También es constante, y a veces abundante, Rizophora mangle L., la frecuente es Conocarpus erectus. El estrato herbáceo la mayor parte de las veces se presenta disperso, pero cuando se desarrolla tiene una cobertura del $50 \%$ y una altura de $20 \mathrm{~cm}$. Lo integran Batis maritima, Suriana maritima L. y plántulas de Avicennia germinans.

Es muy pobre en especies, posee 3,3 como promedio y ninguna es endemismo. Predominan los nanofanerófitos y macrofanerófitos, así como las hojas micrófilas. Existe un extrapófito, Batis maritima, en un $20 \%$ en la cenosis (tab. 1).

Esta fitocenosis se describió solo con Avicennia germinans, que tiene los mayores valores de abundan cia, y Rhizophora mangle, con los menores; ello coincide con el resultado de este estudio, aunque se presentan también Conocarpus erectus y Batis maritima, pero casi siempre con una baja abundancia-dominancia.

\section{Lagunculario racemosae-Rhizophoretum mangle \\ Delgadillo, Peinado, Martínez-Parras \& Alcaraz 1992 [Tabla 6, fig. 3A]}

Es un manglar sobre suelos areno-fangosos, temporalmente inundado. Su areal biogeográfico en Cuba solo es conocido al norte de la laguna del Puerto y al este de la laguna Larga, en cayo Coco.

El estrato arbóreo tiene una cobertura de 60

Tabla 6

Lagunculario racemosae-Rhizophoretum mangle Delgadillo, Peinado, Martínez-Parras \& Alcaraz 1992 (Rhizophoretea mangle, Avicennietalia, Avicennion occidentalis)

\begin{tabular}{|c|c|c|c|c|c|c|c|c|c|c|}
\hline Inventario $\mathrm{n}^{\circ}$ & 14 & \multicolumn{2}{|l|}{15} & 16 & 17 & 18 & 19 & 21 & 22 & 23 \\
\hline Estrato arbóreo E3 (\%) & 90 & \multicolumn{2}{|l|}{60} & 95 & 90 & 95 & 85 & 70 & 90 & 90 \\
\hline Estrato herbáceo E1 (\%) & disp. & \multicolumn{2}{|l|}{ disp. } & disp. & disp. & disp. & disp. & disp. & disp. & disp. \\
\hline $\mathrm{N}^{\circ}$ especies & 8 & \multicolumn{2}{|l|}{6} & 6 & 4 & 5 & 3 & 4 & 5 & 4 \\
\hline \multicolumn{11}{|c|}{ Características de asociación y unidades superiores } \\
\hline Rhizophora mangle $(\mathrm{E} 3,1)$ & 4.1 & 2.1 & 3.1 & 4.1 & \multicolumn{2}{|l|}{4.1} & 5.5 & . & +.1 & 2.1 \\
\hline Conocarpus erectus & 3.1 & 3.1 & 2.1 & 2.1 & \multicolumn{2}{|l|}{3.1} & 4.1 & 2.1 & 3.1 & 3.1 \\
\hline Laguncularia racemosa & +.1 & 2.1 & 2.1 & 3.1 & \multicolumn{2}{|l|}{3.1} & 4.1 & 4.1 & +.1 & 2.1 \\
\hline Avicennia germinans & +.1 & 1.1 & +.1 & +.1 & \multicolumn{2}{|l|}{1.1} & +.1 & +.1 & 2.1 & . \\
\hline \multicolumn{11}{|l|}{ Compañeras } \\
\hline Batis maritima (E1) & r.1 & . & r.1 & . & \multicolumn{2}{|l|}{. } & . & . & . & . \\
\hline Sarcocornia perennis & . & r.2 & . & . & \multicolumn{2}{|l|}{ r.2 } & . & . & . & . \\
\hline Borrichia arborescens & r.1 & . & . & . & \multicolumn{2}{|l|}{. } & . & . & . & . \\
\hline Sesuvium portulacastrum & r.1 & . & . & . & \multicolumn{2}{|l|}{. } & . & . & . & . \\
\hline
\end{tabular}

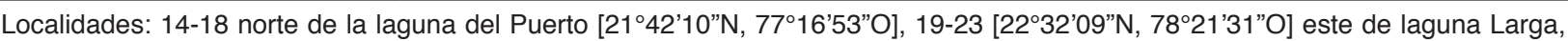
cayo Coco. disp. $=$ disperso 



Figura 3. Fitocenosis de manglares y saladares en las lagunas costeras. A) Lagunculario racemosae-Rhizophoretum mangle al norte de la laguna del Puerto, cayo Coco, B) Batido-Conocarpodetum erecti al norte de la laguna Tortuguilla, cayo Sabinal, C) Lagunculario racemosae-Conocarpodetum erecti al norte de la laguna Tortuguilla, cayo Sabinal, D) Conocarpodetum erecti al oeste de la laguna La Salina, cayo Sabinal, E) Sporobolo virginici-Conocarpodetum erecti al sur de la laguna Tortuguilla, cayo Sabinal, F) Batidetum maritimae en el interior de la laguna Los Caimanes, cayo Sabinal, G) Sarcocornietum perennis al sur de la laguna Tortuguilla, cayo Sabinal, durante la época seca. Mangrove phytocoenosis and salt marshes in coastal lagoons Figure 3. A) Lagunculario racemosae-Rhizophoretum mangle at North of Puerto Lagoon, Coco Cay, B) Batido-Conocarpodetum erecti at North of Tortuguilla Lagoon, Sabinal Cay, C) Lagunculario racemosae-Conocarpodetum erecti, at North of Tortuguilla Lagoon, Sabinal Cay, D) Conocarpodetum erecti at West of La Salina Lagoon, Sabinal Cay, E) Sporobolo virginici-Conocarpodetum erecti at South of Tortuguilla Lagoon, Sabinal Cay, F) Batidetum maritimae inside of Los Caimanes Lagoon, Sabinal Cay, G) Sarcocornietum perennis at South of Tortuguilla Lagoon, Sabinal Cay, during dry season. 
Tabla 7

\begin{tabular}{|c|c|c|c|c|c|c|c|}
\hline \multicolumn{8}{|c|}{$\begin{array}{l}\text { Batido-Conocarpodetum erecti Martínez-Quesada ass. nova } \\
\text { (Rhizophoretea mangle, Combretalia, Batido maritimae-Conocarpodion erecti) }\end{array}$} \\
\hline \multirow{2}{*}{$\begin{array}{ll}\text { Variantes } \\
\text { Inventario } n^{\circ}\end{array}$} & \multicolumn{3}{|c|}{ Sesuvium portulacastrum } & \multicolumn{4}{|c|}{ Fimbristylis cymosa } \\
\hline & 53 & 55 & 56 & 92 & 93 & 94 & 95 \\
\hline Estrato arbóreo E3 (\%) & 85 & 90 & 90 & 55 & 30 & 60 & 60 \\
\hline Estrato herbáceo E1 (\%) & 80 & 25 & 50 & disp. & disp. & disp. & disp. \\
\hline$N^{\circ}$ especies & 6 & 4 & 4 & 9 & 17 & 9 & 5 \\
\hline \multicolumn{8}{|c|}{ Características de asociación y de alianza } \\
\hline Conocarpus erectus $(\mathrm{E} 3,1)$ & 4.4 & 5.5 & 5.5 & 5.5 & 5.5 & 5.5 & 5.5 \\
\hline Batis maritima $(\mathrm{E} 1)$ & 1.2 & 1.2 & 4.4 & +.2 & +.2 & 3.2 & 5.5 \\
\hline \multicolumn{8}{|l|}{ Diferenciales de variantes } \\
\hline Sesuvium portulacastrum (E1) & 4.4 & 1.2 & \multicolumn{2}{|l|}{4.4} & . & . & \\
\hline Rachicallis americana & r.1 & r.1 & \multicolumn{2}{|l|}{+.1} & . & . & \multirow{2}{*}{ r.2 } \\
\hline Fimbristylis cymosa & . & . & . & 4.3 & 4.3 & r.2 & \\
\hline Sesuvium maritimum & . & . & . & +.2 & +.2 & 1.2 & 1.1 \\
\hline Sporobolus virginicus & . & . & . & +.2 & +.2 & 1.2 & 3.3 \\
\hline Broughtonia lindenii & . & . & . & r.1 & r.1 & r.1 & r \\
\hline Selenicereus grandiflorus & . & . & . & . & $\mathrm{r} .1$ & r. 1 & . \\
\hline Sarcocornia perennis (E1) & . & . & . & . & 2.2 & 1.2 & . \\
\hline Evolvulus convolvuloides & . & . & . & $r .1$ & r.1 & . & . \\
\hline Cienfuegosia yucatanensis & . & . & . & +.1 & r.1 & . & \\
\hline Ambrosia hispida & . & . & . & r.1 & +.1 & . & . \\
\hline \multicolumn{8}{|l|}{ Compañeras } \\
\hline Suaeda linearis (E1) & r.1 & . & . & . & . & 1.1 & \\
\hline
\end{tabular}

Otras compañeras: En 53: Salicornia bigellovii +.1, En 93: Iva imbricata +.1, Tabebuia trachycarpa r.1, Sida ciliaris var. ciliaris r.1, Cynanchum caribaeum r.1, Senna chapmanii r.1, Pithecellobium keyense r.1, En 94: Avicennia germinans r.1.

a $95 \%$ y una altura de 5 a $10 \mathrm{~m}$. Las especies constantes son Conacarpus erectus y Rizophora mangle, la más abundantes, Avicennia germinans y Laguncularia racemosa. El estrato herbáceo está disperso; no obstante, se observan Batis maritima y Sarcocornia perennis. Además se aprecian, como parte de la regeneración del bosque, a juveniles de $R$. mangle, A. germinans y L. racemosa.

Es pobre en especies, posee 5 como promedio y ninguna es endemismo. Se presentan los nanofanerófitos y macrofanerófitos, con predominio de las hojas micrófilas. Posee un extrapófito, Batis maritima, y un intrapófito recurrente, Borrichia arborescens, para un $25 \%$ de la cenosis (tab. 1).

Este manglar, donde Rhizophora mangle aparece como especie dominante, ha sido observado en Baja California (México) por Delgadillo et al. (1992) y Peinado et al. (1994), aunque sin Conocarpus erectus entre las especies de mangles, y en general, por la falta de otras especies vasculares que aquí se presentan pero con una baja abundancia. Es coincidente el hecho del abrupto ecotono con otras comunidades, como el bosque siempreverde, al norte de la laguna del Puerto y el matorral xeromorfo costero, al este de la laguna Larga. En Cuba no se había registrado.

\section{Combretalia Cuatrecasas 1958}

\section{Batido-Conocarpodetum erecti Martínez-Quesada} ass. nova

[Tabla 7, holotypus ass. inv. 93; fig. 3B]

Características de asociación: Conocarpus erectus y Batis maritima.

Constituye un manglar sobre suelos arenosos, hipersalinos, que se establece en la parte más alejada de la influencia del agua de las lagunas y de las mareas. Se encuentra al sur de la laguna del Puerto, en cayo Coco y al norte de laguna Tortuguilla, en cayo Sabinal.

El estrato arbóreo tiene una cobertura del 30 a $90 \%$ y una altura de 2 a $4,5 \mathrm{~m}$. La especie constante y muy abundante es Conocarpus erectus. EI estrato herbáceo, cuando se desarrolla, tiene una 
Tabla 8

Lagunculario racemosae-Conocarpodetum erecti

Peinado, Alcaraz \& Delgadillo 1995(Rhizophoretea mangle, Combretalia, Conocarpo-Laguncularion)

\begin{tabular}{|l|l|l|l|l|l|}
\hline Inventario n & 73 & 74 & 75 & 76 & 77 \\
\hline Estrato arbóreo E3 (\%) & 90 & 85 & 90 & 90 & 95 \\
\hline $\mathrm{N}^{\circ}$ especies & 2 & 2 & 2 & 2 & 2 \\
\hline Características de asociación y unidades superiores \\
\hline $\begin{array}{l}\text { Laguncularia racemosa } \\
\text { (E3) }\end{array}$ & 5.5 & 5.5 & 4.4 & 4.4 & 5.5 \\
\hline Conocarpus erectus & +.1 & r.1 & 4.1 & 3.1 & 2.1 \\
\hline
\end{tabular}

Localidades: $73-77$ norte de la laguna Tortuguilla [214' $04^{\prime \prime} \mathrm{N}$, 77¹4'38”], cayo Sabinal.

cobertura del 25 a $80 \%$ y una altura de 10 a $45 \mathrm{~cm}$. La constante y a veces puntualmente abundante es Batis maritima. Menos frecuentes son Broughtonia lindenii (Lindl.) Dressler, Fimbristylis cymosa R. Br., Rachicalis americana (Jacq.) Hitchc., Sesuvium maritimum (L.) L., S. portulacastrum (L.) L., y Sporobolus virginicus, y las ocasionales son Ambrosia hispida Pursh, Cienfuegosia yucatanensis Millsp., Evolvulus convolvuloides (Willd. ex Schult) Stearn., Sarcocornia perennis, Selenicereus grandiflorus (L.) Britton \& Rose, y Suaeda linearis (Elliott) Moq. La sinusia de lianas se compone de Metastelma cubense Griseb. y Selenicereus grandiflorus, y la de epífitas por Broughtonia lindenii.

Presenta dos variantes, de Fimbristylis cymosa que se aprecia en los inventarios 92 a 95 y de Sesuvium portulacastrum en los inventarios 53,55 y 56 . La primera, se diferencia por ser más rica florísticamente al colindar con un bosque siempreverde de donde probablemente proviene un aporte de nutrientes por arrastre de las lluvias.

La asociación es muy pobre en especies, posee 7,7 como promedio y ninguna es endemismo. Se compone principalmente de caméfitos y nanofanerófitos y predominan las hojas leptófilas y micrófilas. Posee cinco especies sinantrópicas: dos extrapófitos, Batis maritima y Selenicereus grandiflorus; un parapófito, Fimbristylis cymosa, un intrapófito pionero, Evolvulus convolvuloides y un intrapófito recurrente, Tabebuia trachycarpa (Griseb.) K.Schum., en un $23,8 \%$ en la cenosis (tab. 1).

Esta fitocenosis se ubica en la alianza Batido maritimae-Conocarpodion erecti, que se describió de las dunas arenosas y conchas marinas (Reyes Domínguez 2006), o solo sobre estas últimas, en la orilla del mar o entre los manglares, o al borde interior de sedimentos cuaternarios; dentro de ella se han descrito dos asociaciones: Distichli spicataeConocarpodetum erecti Reyes Domínguez 2006 y Lycio caroliniani-Conocarpodetum erecti Reyes Domínguez 2006, que difieren de esta nueva por la combinación característica de sus especies y las condiciones ecológicas en las que se desarrolla, las que se pueden contrastar con las de la alianza. Batis maritima integra la combinación característica de dicha alianza, con una alta presencia en los inventarios de la asociación.

\section{Lagunculario racemosae-Conocarpodetum erecti Peinado, Alcaraz \& Delgadillo 1995}

[Tabla 8, fig. 3C]

Es un manglar achaparrado, de poca extensión, que ocupa una franja estrecha al norte de la laguna Tortuguilla, ubicado entre un matorral xeromorfo costero interrumpido por un terraplén, y una comunidad de Rhizophoretum mangle. Está en suelos arenosos, con inundaciones temporales de menor duración (tres a cuatro meses) que los manglares de Avicennietalia.

El estrato arbóreo es el único existente, cubre entre el 85 y $95 \%$ de la superficie y alcanza una altura entre 1,30 y 1,60 m. Laguncularia racemosa es la especie constante y dominante. Además es constante Conocarpus erectus.

Es extremadamente pobre en especies, posee dos, no endémicas, que son macrofanerófitos de hojas micrófilas, no sinantrópicas (tab. 1).

En la literatura fitosociológica se aprecia que existen muy pocas asociaciones descritas con Laguncularia racemosa. Previamente, Delgadillo et al. (1992) y Peinado et al. (1994) se refirieron a Lagunculario racemosae-Rhizophoretum mangle y a Lagunculario racemosae-Avicennietum germinantis, de la costa de Baja California, México. Más recientemente Reyes Domínguez (2006) describió el Laguncularietum racemosae, un manglar que rodea la laguna Birama, en la provincia Las Tunas (Cuba), donde L. racemosa es la especie dominante absoluta. Según Menéndez Carrera et al. (2006) en el ecosistema Sabana-Camagüey no existe ningún bosque con dominancia de $L$. racemosa, y en general se evidencia una pobre representación de la especie en los manglares. Lagunculario racemosae-Conocarpodetum erecti, aunque no abarca una gran extensión superficial, demuestra lo insuficientemente estudiado que está el territorio, pues $L$. racemosa es en ella la especie dominante, por su alta abundancia. En Cuba no se conocía de su presencia. Sintaxonómicamente Lagunculario racemosae-Conocarpodetum erecti se incluyó primero en Lagunculario racemosaeAvicennion germinantis (ahora Avicenion occidentalis), que son manglares desarrollados 
Tabla 9

Conocarpodetum erecti Reyes Domínguez 2003

(Rhizophoretea mangle, Combretalia, Conocarpo-Laguncularion)

\begin{tabular}{|c|c|c|c|c|c|}
\hline Inventario $\mathrm{n}^{\circ}$ & 78 & 79 & 80 & 81 & 82 \\
\hline Estrato arbóreo E3 (\%) & 85 & 90 & 75 & 90 & 90 \\
\hline Estrato herbáceo E1 (\%) & disp. & disp. & disp. & disp. & disp. \\
\hline $\mathrm{N}^{\circ}$ especies & 4 & 4 & 6 & 6 & 6 \\
\hline \multicolumn{6}{|c|}{ Características de asociación y unidades superiores } \\
\hline Conocarpus erectus E3 & 5.1 & 5.1 & 5.1 & 5.1 & 5.1 \\
\hline \multicolumn{6}{|l|}{ Compañeras } \\
\hline Suriana maritima E1 & +.1 & +.1 & +.1 & r.1 & r.1 \\
\hline Lantana involucrata & +.1 & r.1 & +.1 & 2.1 & r.1 \\
\hline Casasia clusiifolia E3,1 & r.1 & . & r.1 & . & +.1 \\
\hline Erithalis fruticosa $\mathrm{E} 1$ & . & r.1 & r. 1 & r. 1 & r. 1 \\
\hline Ernodea littoralis & . & . & . & r.1 & r.1 \\
\hline
\end{tabular}

Otras compañeras: En 80: Rachicalis americana r.1, En 81: Jacquemontia havanensis.

Localidades: $78-82$ norte de la laguna Tortuguilla [2141'09”N, $77^{\circ} 13^{\prime} 44^{\prime \prime O}$ ], cayo Sabinal. disp.: disperso

Tabla 10

\begin{tabular}{|c|c|c|c|c|c|c|c|c|c|}
\hline \multicolumn{10}{|c|}{$\begin{array}{l}\text { Sporobolo virginici-Conocarpodetum erecti Martínez-Quesada } 2014 \\
\text { (Rhizophoretea mangle, Combretalia, Conocarpo-Laguncularion) }\end{array}$} \\
\hline Inventario $\mathrm{n}^{\circ}$ & 83 & 84 & 85 & 86 & 87 & 88 & 89 & 90 & 91 \\
\hline Estrato arbóreo E3 (\%) & 85 & 85 & 80 & 85 & 90 & 85 & 90 & 80 & 90 \\
\hline Estrato herbáceo E1 (\%) & 50 & disp. & 50 & 60 & 90 & 75 & 70 & 85 & 95 \\
\hline $\mathrm{N}^{\circ}$ especies & 7 & 5 & 7 & 7 & 5 & 4 & 5 & 7 & 6 \\
\hline \multicolumn{10}{|c|}{ Características de asociación y unidades superiores } \\
\hline Conocarpus erectus (E3) & 5.1 & 3.1 & 5.1 & 5.1 & 5.1 & 5.1 & 5.1 & 4.1 & 4.1 \\
\hline Laguncularia racemosa & . & . & . & . & . & . & r.1 & +.1 & +.1 \\
\hline Avicennia germinans (E1) & & . & & . & r. 1 & 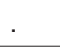 & & r.1 & r.1 \\
\hline \multicolumn{10}{|l|}{ Compañeras } \\
\hline Sporobolus virginicus (E1) & 2.2 & 2.2 & 3.2 & 4.2 & 4.5 & +.2 & 3.4 & 4.5 & 3.4 \\
\hline Batis maritima & . & . & +.2 & +.2 & 1.2 & 1.2 & 1.2 & +.2 & +.2 \\
\hline Sarcocornia perennis & . & . & 2.2 & +.2 & 1.2 & +.2 & . & 1.2 & +.2 \\
\hline Jacquinia keyensis & r.1 & r.1 & r.1 & +.1 & . & . & . & . & . \\
\hline Pilosocereus royenii (E3) & r.1 & r.1 & r. 1 & . & . & . & . & . & . \\
\hline Sesuvium maritimum & r.1 & . & +.2 & 2.1 & . & . & . & . & . \\
\hline Sesuvium portulacastrum & . & . & . & . & . & . & 1.2 & +.2 & . \\
\hline Pithecellobium mucronatum & r.1 & +.1 & . & . & . & . & . & . & . \\
\hline Tillandsia paucifolia & r.1 & . & . & +.2 & . & . & . & . & . \\
\hline
\end{tabular}

Otras compañeras: En 89: Bucida molinetii r.1, En: 90 Sideroxylon horridum r.1, En 91: Distichlis spicata 2.2.

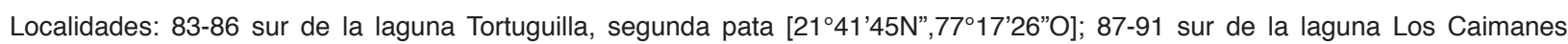
[21 $44^{\prime} 59^{\prime \prime} \mathrm{N}, 77^{\circ} 21^{\prime} 33^{\prime \prime} \mathrm{O}$ ], cayo Sabinal. disp.: disperso 
en la zona de pleamar (Peinado et al., 1995); sin embargo, en un trabajo posterior Cortés-Castillo \& Rangel-Ch. (2011) la transfirieron a Lagunculario racemosae-Rhizophorion mangle (ahora Rhizophorion occidentalis), que son manglares que se desarrollan en la parte más baja de las mareas, por los valores de la cobertura de L. racemosa, en iguales condiciones ecológicas que las observadas en este estudio. Se considera que la LaguncularioConocarpodetum debe ser ubicada en ConocarpoLaguncularion (Sin. Lagunculario racemosaeAvicennion germinantis), ya que es un manglar de muy baja altura, estacionalmente inundado, casi siempre alejado de la influencia directa de las mareas. Por ello los valores de salinidad en el suelo deben fluctuar anualmente. Florísticamente, la presencia constante de Conacarpus erectus, aunque no siempre con altos valores de su abundancia-dominancia, también justifica su inclusión en esta alianza.

Conocarpodetum erecti Reyes Domínguez in Reyes Domínguez \& Acosta Cantillo 2003

[Tabla 9, fig. 3D]

Es un manglar sobre suelos arenosos y pavimento carstificado con parches de arena fangosa.

$\mathrm{Su}$ areal biogeográfico es el sur de cayo Anclitas, archipiélago Jardines de la Reina, parte central de Cuba, así como la costa sur de Santiago de Cuba y norte de Guantánamo, parte oriental del país. Se observó al norte de la laguna Tortuguilla y al oeste de la laguna La Salina.

El estrato arbóreo cubre del 75 al $90 \%$ y tiene una altura que va desde 3 a 4,5 $\mathrm{m}$. La especie dominante absoluta es Conocarpus erectus. El estrato herbáceo está disperso. Se observan
Casasia clusiifolia (Jacq.) Urb., Erithalis fruticosa L., Ernodea littoralis Sw., Lantana involucrata L., y Suriana maritima, y las especies constantes son Ernodea littoralis, L. involucrata, y Suriana maritima, mientras que Casasia clusiifolia es frecuente.

Es muy pobre en especies, posee 6 como promedio y ninguna es endemismo. Se compone en su mayoría de los nanofanerófitos y predominan las hojas nanófilas. Presenta un intrapófito recurrente, Lantana involucrata, y un intrapófito pionero, Erithalis fruticosa, en un $28,5 \%$ en la cenosis (tab. 1).

Reyes Domínguez (Reyes Domínguez \& Acosta Cantillo, 2003) describió el Conocarpodetum erecti como un manglar puro de Conocarpus erectus y sin un estrato herbáceo. En cayo Sabinal se presenta un bosque, donde el estrato arbóreo está dominado por esa especie y aparecen algunas herbáceas que tienen una baja abundancia-dominancia, como ocurre en cayo Anclitas (Martínez-Quesada, 2014). Esa es la razón por la cual no se considera una asociación distinta.

\section{Sporobolo virginici-Conocarpodetum erecti} Martínez-Quesada 2014

[Tabla 10, fig. 3E]

Constituye un manglar sobre pavimento carstificado, liso o en forma de lapiaz, temporalmente inundado. Su areal biogeográfico conocido es el suroeste de Cayo Anclitas. Se observó al sur de la laguna Los Caimanes, sur de la laguna Tortuguilla, y sur de laguna La Salina, en cayo Sabinal.

El estrato arbóreo posee una cobertura del 80 a $90 \%$ y una altura de 2 a $5 \mathrm{~m}$. La constante y muy abundante es Conocarpus erectus.

Tabla 11

Batidetum maritimae Samek 1973

(Batido-Sarcocornietea, Batido -Sarcocornietalia, Fimbrystilo-Sarcocornion perennis)

\begin{tabular}{|c|c|c|c|c|c|c|c|c|}
\hline Inventario $\mathrm{n}^{\circ}$ & 40 & 41 & 42 & 43 & 44 & 96 & 97 & 98 \\
\hline Estrato herbáceo E1 (\%) & 95 & 90 & 90 & 90 & 85 & 85 & 50 & 60 \\
\hline $\mathrm{N}^{\circ}$ especies & 2 & 2 & 1 & 1 & 1 & 4 & 4 & 5 \\
\hline \multicolumn{9}{|c|}{ Característica de asociación y unidades superiores } \\
\hline Batis maritima $(\mathrm{E} 1)$ & 5.5 & 5.5 & 5.5 & 5.5 & 5.5 & 4.5 & 4.5 & 4.5 \\
\hline Sesuvium portulacastrum & r.1 & r.1 & . & . & . & 1.2 & +.2 & 2.1 \\
\hline Salicornia bigelovi & . & . & . & . & . & +.1 & +.1 & r.1 \\
\hline \multicolumn{9}{|l|}{ Compañeras } \\
\hline Avicennia germinans & . & . & . & . & . & +.1 & +.1 & +.1 \\
\hline Sarcocornia perennis & . & . & . & . & . & . & . & +.2 \\
\hline
\end{tabular}

Localidades: $40-44$ interior de la laguna Los Caimanes, por el norte $\left[21^{\circ} 45^{\prime} 15 N^{\prime \prime}, 77^{\circ} 21^{\prime} 06^{\prime \prime} \mathrm{O}\right]$, $96-98$ sur de laguna Tortuguilla [214' $45^{\prime \prime} \mathrm{N}, 77^{\circ} 19^{\prime} 42^{\prime \prime} \mathrm{O}$ ], cayo Sabinal. 
Son menos frecuentes, Avicennia germinans, Laguncularia racemosa y Pilosocereus royenii (L.) Byles \& G.D. Rowley. El estrato herbáceo, generalmente presente, cubre entre un 50 y $95 \%$ de la superficie y tiene una altura de 20 a $40 \mathrm{~cm}$. Es constante Sporobolus virginicus, frecuentes son Batis maritima y Sarcocornia perennis, como menos frecuentes están Jacquinia keyensis Mez. y Sesuvium maritimum, y las ocasionales son Pithecellobium mucronatum Coker. y Sesuvium portulacastrum. La sinusia de epífitas solo está representada por Tillandsia paucifolia Baker., que es una especie ocasional.

Con un promedio de 6,4 especies no endémicas, predominan los nanofanerófitos de hojas micrófilas. Posee un extrapófito, Batis maritima en un 6,6\% en la cenosis (tab. 1).

Sporobolo virginici-Conocarpodetum erecti se había descrito a partir de seis inventarios en cayo Anclitas, sobre arenas calcáreas, eólicomarinas y con muy pocas especies (MartínezQuesada, 2014). En cayo Sabinal se observó sobre pavimento carstificado que puede inundarse temporalmente y aunque se encontraron también en el estrato arbóreo a Avicennia germinans y Laguncularia racemosa, son menos frecuentes y tienen baja abundancia-dominancia. Además de Sporobolus virginicus, en el estrato herbáceo, se presentan muchas más especies que en cayo Anclitas. Esta asociación al parecer puede ser más o menos rica florísticamente de acuerdo con las condiciones abióticas y la capacidad de las especies de adaptarse a ellas, o de su cercanía a otras formaciones vegetales, como el bosque siempreverde micrófilo de los cayos, que muchas veces colinda con las lagunas costeras siendo la causa por la cual crecen em esta fitocenosis Bucida molinetii (M. Gómez) Alwan \& Stace, Jacquinia keyensis, Pilosocereus royenii, Pithecellobium mucronatum. Como Conocarpus erectus y Sporobolus virginicus son las dominantes y de más alta constancia, se considera que es la misma asociación, aunque se encuentre en zonas de pavimento carstificado. Por observaciones personales, en otras partes del cayo, cuando está alejada de las lagunas, el suelo también puede ser arenoso con parches pavimentados.

\section{Batido maritimae-Sarcocornietalia ambiguae Borhidi} 1996

\section{Batidetum maritimae Samek 1973}

[Tabla 11, fig. 3F]

Corresponde a una vegetación halófila estacionalmente inundado. Se describió de la costa norte de la provincia La Habana, en la parte central de Cuba, pero debe estar más extendida por todo el archipiélago. Está ubicada en el interior de la laguna Los Caimanes, por el norte, donde constituye un gran parche de Batis maritima, casi puro, que se desarrolla sobre un suelo arenoso, muy húmedo y también sobre pavimento carstificado con parches de arena, al sur de las lagunas Tortuguilla y La Salina, ambas

Tabla 12

\begin{tabular}{|c|c|c|c|c|c|}
\hline \multicolumn{6}{|c|}{$\begin{array}{c}\text { Sarcocornietum perennis Samek } 1973 \\
\text { (Batido-Sarcocornietea, Batido-Sarcocornietalia, } \\
\text { Fimbrystilo-Sarcocornion perennis) }\end{array}$} \\
\hline Inventario $\mathrm{n}^{\circ}$ & 99 & 100 & 101 & 102 & 103 \\
\hline Estrato herbáceo E1 (\%) & 50 & 90 & 90 & 85 & 90 \\
\hline $\mathrm{N}^{\circ}$ especies & 7 & 6 & 5 & 5 & 5 \\
\hline \multicolumn{6}{|c|}{ Características de asociación y unidades superiores } \\
\hline Sarcocornia perennis (E1) & 1.4 & 3.4 & 5.5 & 4.4 & 4.4 \\
\hline Suaeda linearis & +.1 & +.1 & +.1 & +.1 & 1.1 \\
\hline Fimbristylis cymosa & +.2 & +.2 & . & & . \\
\hline \multicolumn{6}{|l|}{ Compañeras } \\
\hline Conocarpus erectus (E1) & 4.1 & 2.1 & 1.1 & 1.1 & 1.1 \\
\hline Sesuvium maritimum & +.2 & +.2 & r.2 & +.2 & 1.2 \\
\hline Sporobolus virginicus & 3.2 & 2.2 & 2.2 & 2.2 & 2.2 \\
\hline Frimbristylis ferruginea & +.2 & . & . & . & . \\
\hline
\end{tabular}

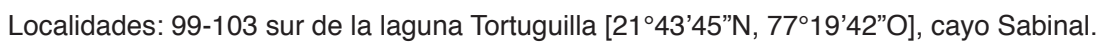




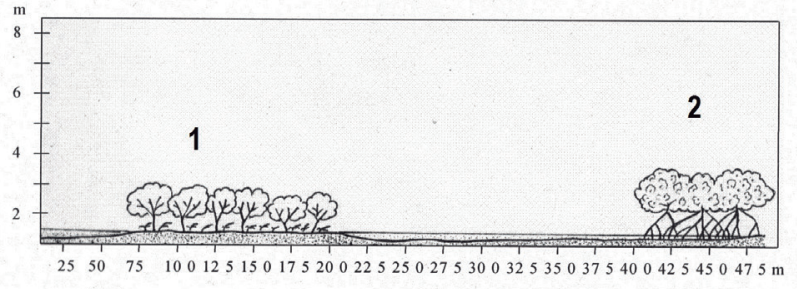

A

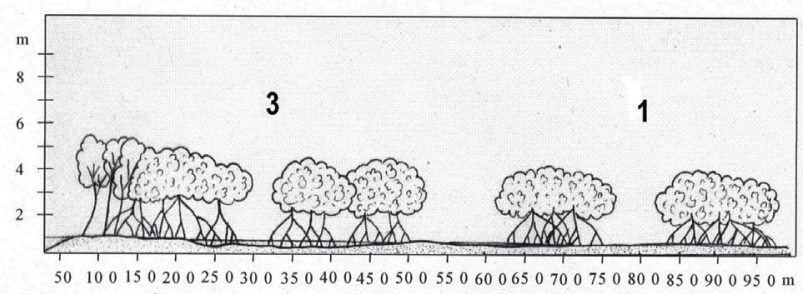

B

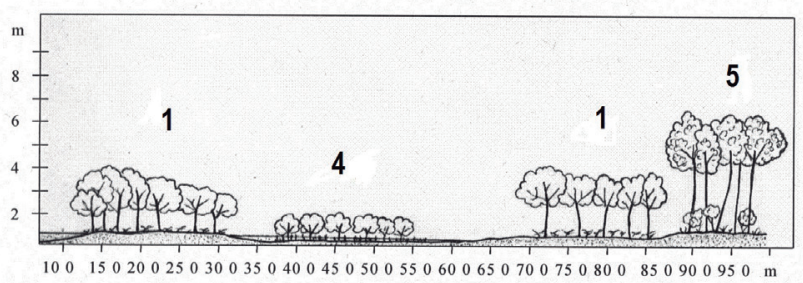

C

Figura 4. Zonación de las fitocenosis de manglar. A) Laguna Las Coloradas, B) Laguna Larga, extremo oriental, y C) Laguna Tiburón. 1- Batido-Avicennietum germinantis, 2- Rhizophoretum mangle, 3- Lagunculario racemosae-Rhizophoretum mangle, 4- Avicennietum germinantis, 5- Bosque semidecíduo micrófilo. Figure 4. Mangrove phytocoenosis zoning. A) Las Coloradas Lagoon, B) Larga Lagoon, west part, and C) Tiburón Lagoon.

en cayo Sabinal.

El estrato herbáceo posee una cobertura del 85 a $90 \%$ y una altura de 35 a $50 \mathrm{~cm}$. La especie constante y muy abundante es Batis marítima, menos frecuente se observa a Sesuvium portulacastrum (L.) L. Se encuentran también, sobre pavimento carstificado, Avicennia germinans, con $40 \mathrm{~cm}$ de altura, Salicornia bigelovii Torr. a Sarcocornia perennis, todas con baja abundanciadominancia.

Es muy pobre en especies, posee 2,5 como promedio y ninguna es endemismo. Predominan los nanofanerófitos, con hojas leptófilas, nanófilas y micrófilas. Existe un extrapófito en la cenosis, Batis maritima, en un $20 \%$ (tab. 1).

\section{Sarcocornietum perennis Samek 1973}

[Tabla 12, fig. 3G]

Representa un herbazal halófilo estacionalmente inundado que se desarrolla sobre pavimento carstificado y parches de arena, en el
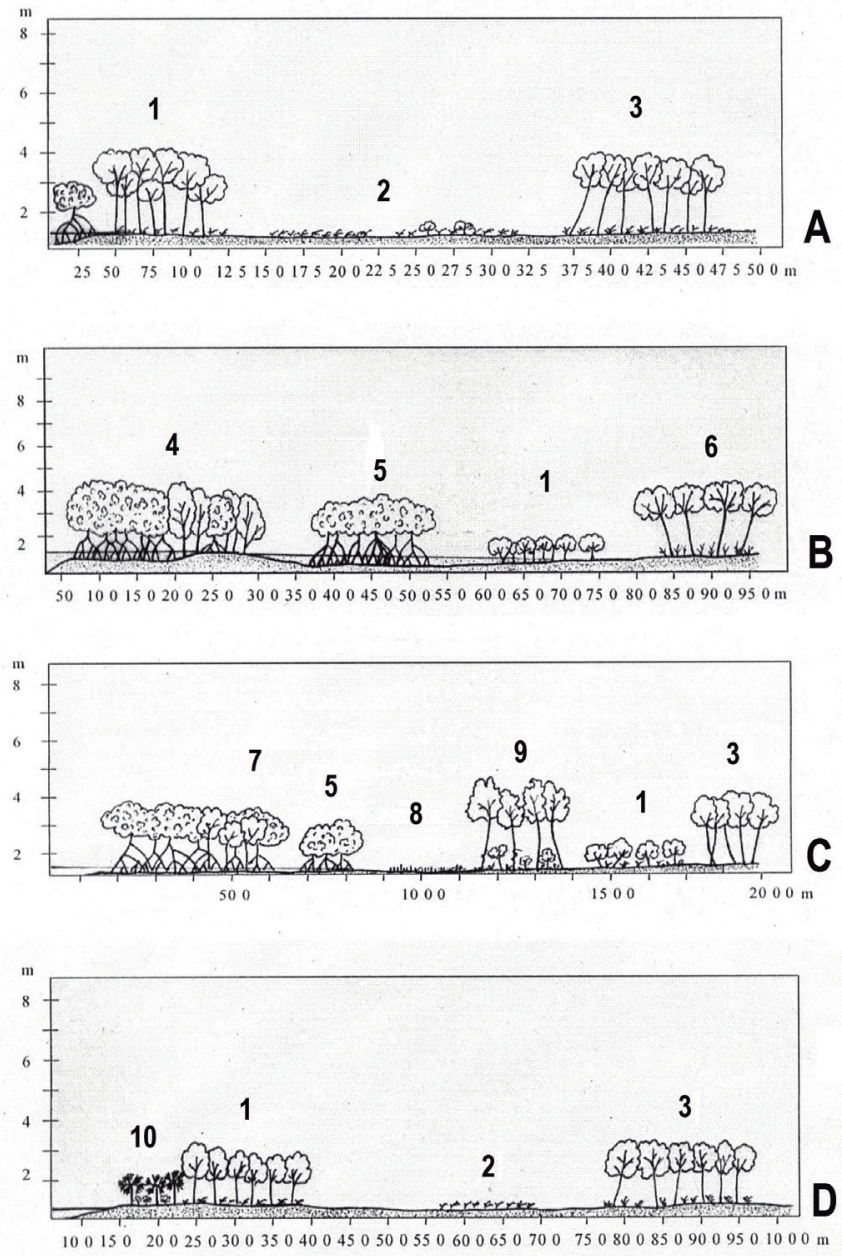

Figura 5. Zonación de las fitocenosis de manglar. A) Laguna Los Caimanes, B) Laguna del Puerto, C) Laguna Tortuguilla, D) Laguna La Salina. 1- Batido-Avicennietum germinantis, 2- Batidetum maritimae, 3- Sporobolo virginici-Conocarpodetum erecti, 4- Lagunculario racemosae-Rhizophoretum mangle, 5- Rhizophoretum mangle, 6- Batido-Conocarpodetum erecti, 7- Avicennio germinantis-Rhizophoretum mangle, 8- Sarcocornietum perennis, 9- Bosque siempreverde micrófilo, 10-Matorral xeromorfo costero. Figure 5. Mangrove phytocoenosis zoning. A) Los Caimanes Lagoon, B) El Puerto Lagoon, C) Tortuguilla Lagoon, D) La Salina Lagoon.

interior de las lagunas. Se describió de la costa norte de Alamar, provincia La Habana, en la parte central de Cuba, pero debe estar más extendida por todo el archipiélago. Se observó por el sur de la laguna Tortuguilla, en cayo Sabinal.

El estrato herbáceo posee una cobertura del 50 a $90 \%$ y la altura oscila entre 25 y 35 $\mathrm{cm}$. La constante y generalmente abundante es Sarcocornia perennis. Otras especies constantes son Conocarpus erectus, Sesuvium maritimum, Sporobolus virginicus, y Suaeda linearis. Menos frecuente es Fimbristylis cymosa. Los individuos de Conocarpus erectus alcanzan los $50 \mathrm{~cm}$, por lo 
que pueden considerarse como emergentes.

Presenta 5,6 especies como promedio. Predominan los caméfitos y los nanofanerófitos y las hojas son leptófilas, nanófilas y micrófilas (tab. 1).

Muy relacionada con Sarcocornietum perennis está Fimbristylo spathaceae-Sarcocornietum perennis. Ambas asociaciones tienen una composición florística y condiciones ecológicas similares. Debido a que estos sintaxones no se diferencian significativamente deben considerarse como uno solo bajo el nombre Sarcocornietum perennis de acuerdo con el art. $18 \mathrm{c}$ del Código Internacinal de Nomenclatura Fitocenológica (Weber et al., 2000).

\section{Zonación de las comunidades}

En general las comunidades estudiadas no exhiben siempre la misma zonación. Por el norte de algunas lagunas, la primera zona es ocupada por Rhizophoretum mangle, alrededor del canal o boca de entrada, a veces también se extiende unos metros más allá del mismo; no obstante, puede aparecer en el interior del cuerpo de agua, en forma de parches más o menos grandes y ocupando la primera franja al sur, como en la lagunas Las Coloradas y Larga, en cayo Coco (fig. 4 A y B). En otras lagunas, en lugar de Rhizophoretum mangle se hallan por el norte el Lagunculario racemosaeRhizophoretum mangle o Batido-Avicennietum germinantis (fig. 4A-C, fig. 5A y B).

Hacia el interior de las lagunas se observan Avicennietum germinantis, Batidetum maritimae y Sarcocornietum perennis (fig. 4C, fig. 5A y C); mientras que por el sur muchas veces se encuentra el Sporobolo virginici-Conocarpodetum erecti o Batido-Conocarpodetum erecti (fig. 5 A-D). La zonación en la laguna Tortuguilla, en cayo Sabinal, es realmente variable (fig. 5C). Por el norte lo mismo Rhizophoretum mangle, Avicennio germinantis-Rhizophoretum mangle, BatidoConocarpodetum erecti, Batido-Avicennietum germinantis que Lagunculario racemosaeConocarpodetum erecti pueden ocupar la primera zona y más hacia el interior aparecen, como en otras lagunas, Batidetum maritimae y Sarcocornietum perennis. En el sur, pueden verse otra vez BatidoAvicennietum germinantis y Sporobolo virginiciConocarpodetum erecti, que casi siempre ocupa la parte más externa de las lagunas.

El hecho de que las bandas de las comunidades no tengan un patrón fijo en las lagunas tiene mucha relación con los procesos de sedimentación, el desarrollo de suelos arenosos, la pavimentación del carso y la inundación, básicamente dependen del tipo de sustrato y la estacionalidad. Tolinson (1986) y McKee (1993) plantearon que la zonación está sujeta a la topografía local y se relaciona con la composición y estabilidad del sedimento. Otros autores como Boto \& Wellington (1984), Nickerson \& Thibodeau (1985), López-Portillo \& Ezcurra, 1989a, 1989b) y Jiménez \& Sauter (1991) manifestaron que los patrones de distribución de especies en los manglares se han correlacionado previamente con varios factores, como la salinidad, $\mathrm{pH}$ del suelo y concentraciones de ácido sulfhídrico en el agua intersticial, lo cual no se pudo constatar en este estudio, pero pudiera ser la causa de las diferencias florísticas entre las fitocenosis que se presentan en suelos morfológicamente parecidos o iguales. También Pannier \& Fraíno de Pannier (1989) consideran que la zonación del manglar en franjas dominadas por determinadas especies es una consecuencia de la eficiencia metabólica propia de la especie, lo cual se puede apreciar en las especies de mangles dominantes por su abundancia-dominancia.

\section{Tipos biológicos y de hojas}

En las fitocenosis se aprecia que el tipo biológico predominante es el nanofanerófito $(30,7 \%)$, aunque el microfanerófito y el caméfito son también importantes $(17,9 \%)$. Las hojas son mayormente micrófilas (40\%), seguido por las nanófilas y leptófilas (23,3\%), (tab. 1).

Menéndez Carrera et al. (1987) encontraron en los manglares de la cayería al norte de la provincia Matanzas, en el ecosistema Sabana-Camagüey, un predominio de árboles de mediana altura y de hojas notófilas. Posteriormente, Menéndez Carrera et al. (2006) expusieron que la vegetación de manglar, en correspondencia con la gran diversidad de condiciones ecológicas existentes en las costas, presenta variantes fisonómicas, y que de acuerdo a ello será la altura alcanzada. Por su parte, Vilamajó \& Vales (2006), con relación al área foliar de los mangles cubanos, hallaron un $75 \%$ de hojas notófilas, lo cual relacionaron directamente con la xeromorfía y las condiciones del hábitat. El análisis está basado mayormente en especies de mangles, por lo que difiere de los resultados obtenidos aquí; sin embargo, demuestra que el tamaño de las plantas y el área foliar están estrechamente relacionados con determinados factores abióticos que interactúan entre sí, limitando su desarrollo, y que en este estudio bien pueden ser la topografía del terreno, escorrentía, salinidad y disponibilidad de agua dulce.

\section{Sinantropismo}

Las especies sinantrópicas son diez, para 
un $32,2 \%$ del total de las inventariadas. De ellas, los apófitos representan el $90 \%$ y el resto está constituido por un parapófito. Dentro de los apófitos, los intrapófitos recurrentes y los intrapófitos pioneros representan el $77,7 \%$ y los extrapófitos el 22,2\% (tab. 1).

Los apófitos, es decir las especies sinantrópicas de origen nativo, son mayoría, lo cual reafirma el carácter de apofitismo en la flora sinantrópica de Cuba (Ricardo et al., 1990, 1995), siendo las especies dominantes, cuyo número de individuos pueden reinvadir su hábitat bajo un fuerte impacto antrópico (intrapófitos recurrentes), como Borrichia arborescens, Lantana involucrata y Tabebuia trachycarpa y otro grupo de ellas integrado por especies pioneras cuyos individuos se incrementarían de forma explosiva bajo cualquier alteración (intrapófitos pioneros), como Erithalis fruticosa, Evolvulus convolvuloides y Jacquemontia havanensis. Por otra parte, existe un grupo minoritario compuesto por especies que excederían su hábitat (extrapófitos), como Batis maritima y Selenicereus grandiflorus. Esto podría provocar cambios en la abundancia de las especies si estas comunidades fueran seriamente alteradas por causas antrópicas, pero sobre todo significa que su recuperación sería a partir de especies nativas, ya que no existen antropófitos o exóticas, que además no se podrían adaptar a las condiciones ecológicas imperantes. Un análisis del fenómeno en las formaciones vegetales pone de manifiesto que en los saladares la incidencia de especies de este tipo es muy baja o no existen. Solo Batis maritima se presenta como tal y en los manglares los apófitos predominan, principalmente los extrapófitos, representados por la propia B. maritima. Similares resultados obtuvieron Romero-Jiménez et al. (2015) en cayo Las Brujas, archipiélago Sabana-Camagüey, Villa Clara, aunque en el manglar ellos encontraron mayor cantidad de parapófitos que apófitos, por la influencia de las zonas de ecotono.

\section{ESQUEMA SINTAXONÓMICO}

RHIZOPHORETEA MANGLE O. Bolòs, Cervi \& Hatschbach 1991

[Sin.: Rhizophoro mangle-Laguncularietea racemosae Peinado, Alcaraz \& Delgadillo 1995; Rhizophoro-Avicennietea germinantis Borhidi \& Del-Risco in Borhidi 1996]

+ Rhizophoretalia Cuatrecasas 1958 [Sin.: Rhizophoretalia mangle Bolòs, Cervi \& Hatschbach 1991; Rhizophoro mangle-Laguncularietalia racemosae Peinado, Alcaraz \& Delgadillo 1995]

${ }^{*}$ Rhizophorion occidentalis Cuatrecasas 1958 [Sin.:
Rhizophorion mangle Bolòs, Cervi \& Hatschbach 1991, Lagunculario racemosae-Rhizophorion mangle Peinado, Alcaraz \& Delgadillo 1995]

1. Rhizophoretum mangle Cuatrecasas 1958

[Manglar sobre sedimentos arenosos, permanentemente inundado]

+ Avicennietalia Cuatrecasas 1958

*Avicennion occidentalis Cuatrecasas 1958 [Sin.: Lagunculario racemosae-Avicennion germinantis Peinado, Alcaraz \& Delgadillo 1995]

2. Batido-Avicennietum germinantis Borhidi \& Del Risco in Borhidi 1991 (Sin.: Batido maritimaeAvicennietum germinantis Peinado, Alcaraz \& Delgadillo 1995)

[Manglar sobre pavimento carstificado o suelos areno-fangosos, temporalmente inundado]

3. Avicennietum germinantis Reyes Domínguez \& Acosta Cantillo 2003

[manglar sobre suelos fangosos, sulfurosos, temporalmente inundado]

4. Avicennio germinantis-Rhizophoretum mangle Reyes Domínguez \& Acosta

Cantillo 2006 in Reyes Domínguez 2006

[Manglar sobre suelos areno-fangosos, temporalmente inundado]

5. Lagunculario racemosae-Rhizophoretum mangle Peinado et al. 1992

[Manglar sobre suelos areno-fangosos, temporalmente inundado]

+ Combretalia Cuatrecasas 1958

*Conocarpo-Laguncularion Cuatrecasas ex Borhidi 1996 [Sin.: Lagunculario racemosae-Avicennion germinantis Peinado, Alcaraz \& Delgadillo 1995]

6. Sporobolo virginici-Conocarpodetum erecti Martínez-Quesada 2014

[Manglar sobre pavimento carstificado, temporalmente inundado]

*Batido maritimae-Conocarpodion erectae Reyes 2006

7. Batido-Conocarpodetum erecti MartínezQuesada ass. nova

[Manglar sobre suelos arenosos, hipersalinos]

- var. con Fimbristylis cymosa

- var. con Sesuvium portulacastrum

8. Lagunculario racemosae-Conocarpodetum erecti Peinado, Alcaraz \& Delgadillo 1995

[Manglar sobre suelos arenosos, temporalmente inundado]

9. Conocarpodetum erecti Reyes Domínguez 2003 in Reyes Domínguez \& Acosta Cantillo 2003

[Manglar sobre suelos arenosos y pavimento carstificado]

BATIDO-SARCOCORNIETEA AMBIGUAE Borhidi 1996, nom. mut. propos. Galán de

Mera, Linares Perea, Campos \& Vicente Orellana 
2009 [Sin.: Batidi-Salicornietea

Knapp 1964, nom. inv. art. 7, Batido-Salicornietea Borhidi 1991, nom. inv. art. 5]

+ Batido-Sacocornietalia ambiguae Borhidi 1996, nom. mut. propos. Martínez-Quesada 2014 [Sin.: Batido-Salicornietalia ambiguae Borhidi 1996]

${ }^{*}$ Fimbristylo-Sarcocornion perennis Borhidi 1996, nom mut. propos. Martínez-Quesada 2014

10. Batidetum maritimae Samek 1973

[Vegetación halófila, sobre suelos arenosos húmedos y pavimento carstificado con parches de arena, estacionalmente inundada]

11. Sarcocornietum perennis Samek 1973 nom. mut. propos. Galán de Mera, García Fuentes \& Martínez-Quesada 2015 [Sin.: Salicornietum perennis Samek 1973, Fimbristylo spathaceaeSarcocornietum perennis Borhidi in Borhidi, Muñiz \& Del Risco in Acta Bot. Hung. 29: 375 (1983)]

[Vegetación halófila, sobre pavimento carstificado y parches arenosos, estacionalmente inundada]

\section{Agradecimientos}

Este trabajo se realizó en el marco del proyecto "La diversidad biológica de las lagunas costeras del Archipiélago Sabana-Camagüey en el contexto del Cambio Climático Global. Pautas para su manejo y conservación", financiado por el programa científicotécnico "Uso sostenible de la Diversidad Biológica en Cuba", de la Agencia de Medio Ambiente. A los revisores anónimos y editor, por sus valiosas y acertadas sugerencias, las que permitieron mejorar sustancialmente los resultados obtenidos. A Isis Hernández Sosa por la preparación del mapa. Dedico el trabajo a la memoria de Leda Menéndez Carrera, distinguida ecóloga e incansable investigadora de los manglares cubanos.

\section{Referencias}

Batista, L.M., González, R., Zúñiga, A., Matos, F., Hernández, L. \& González, D. (2006). Ecosistemas costeros. Biodiversidad y gestión de recursos naturales. Ciego de Ávila: Cujae.

Borhidi, A. (1976). Fundamentos de Geobotánica en Cuba. Budapest: Tesis Doctoral en Ciencias Biológicas.

Borhidi, A. (1991). Phytogeography and Vegetation Ecology of Cuba. Budapest: Akadémiai Kiadó,

Borhidi, A. (1996). Phytogeography and Vegetation Ecology of Cuba (2nd. ed.). Budapest: Akadémiai Kiadó.

Boto, K.G. \& Wellington, J.T. (1984). Soil characteristics and nutrient status in a northern Australian mangrove forest. Estuaries and Coasts, 7, 61-69. https://doi. org/10.2307/1351957

Braun Blanquet, J. (1979). Fitosociología. Bases para el estudio de las comunidades vegetales. Madrid: Blume Ediciones.

Campos Mazorra, A. \& Vigón del Busto, F.A. (1989).
Insolación anual. 1: 3000 000. Nuevo Atlas Nacional de Cuba. La Habana: Instituto de Geografía de la Academia de Ciencias de Cuba e Instituto Cubano de Geodesia y Cartografía.

Capote, R. \& Berazaín, R. (1984). Clasificación de las formaciones vegetales de Cuba. Revista del Jardín Botánico Nacional, Universidad de la Habana, 5(2), 27-75.

Cortés-Castillo, D.V. \& Rangel-Ch., O. (2011). Los bosques de mangle en un gradiente de salinidad en la Bahía de Cispatá-Boca Tinajones, Departamento de Córdoba, Colombia. Caldasia, 33(1), 155-176.

Crespo González, S.E. (1989). Evaporación media anual. 1: 2000 000. Nuevo Atlas Nacional de Cuba. La Habana: Instituto de Geografía de la Academia de Ciencias de Cuba e Instituto Cubano de Geodesia y Cartografía.

Delgadillo, J., Peinado, M., Martínez-Parras, J.M. \& Alcaraz, F. (1992). Análisis fitosociológico de los saladares y manglares de Baja California, México. Acta Botanica Mexicana, 19, 1-35.

Galán de Mera, A., Hagen, M.A. \& Vicente Orellana, J.A. (1999). Aerophyte, a new life form in Raunkiaer's classification? Journal of Vegetation Science, 10, 6568. doi: $10.2307 / 3237161$

Galán de Mera, A. \& Vicente Orellana, J.A. (2006). Aproximación al esquema sintaxonómico de la vegetación de la región del Caribe y América del Sur. Anales de Biología, 28, 3-27.

Galán de Mera, A., García-Fuentes, A. \& MartínezQuesada, E. (2015). Nota sintaxonómica sobre Sarcocornietum perennis en Cuba e Islas Canarias. Acta Botanica Malacitana, 40, 235-237. http://dx.doi. org/10.24310/Actabotanicaabmabm.v40i0.2504. g2302

Hernández Santana, J.R., Díaz Díaz, L.L. (1989). Geomorfología (tierra firme). In Chiappy Jhones, C., E. Pérez Carreras, N. Enríquez Salgueiro \& R. Vandama (Eds.), Estudio de los grupos insulares y zonas litorales del archipiélago cubano con fines turísticos. Cayo Sabinal-Playa Santa Lucía (pp. 94). La Habana: Centro de Investigaciones de Geodesia, Cartografía y Teledetección del Instituto Cubano de Geodesia y Cartografía.

Hernández Santana, J.R. \& Díaz Díaz, L.L. (1989). Caracterizacióngeográficadelazonayfundamentación teórico metodológica de las investigaciones. In Chiappy Jhones, C., E. Pérez Carreras, N. Enríquez Salgueiro \& R. Vandama (Eds.), Estudio de los grupos insulares y zonas litorales del archipiélago cubano con fines turísticos. Cayo Sabinal-Playa Santa Lucía (pp. 12). La Habana: Centro de Investigaciones de Geodesia, Cartografía y Teledetección del Instituto Cubano de Geodesia y Cartografía.

Izquierdo Ramos, A. (1989). Precipitación media anual 1964-1983. 1200000. Nuevo Atlas Nacional de Cuba. La Habana: Instituto de Geografía de la Academia de Ciencias de Cuba e Instituto Cubano de Geodesia y Cartografía.

Jiménez, J.A. \& Sauter, K. (1991). Structure and dynamics of mangrove forests along a flooding gradient. Estuaries and Coasts, 14, 49-56. https://doi. 
org/10.2307/1351981

Lapinel Pedroso, B. (1989). Temperatura media anual del aire. 1: 2000000. Nuevo Atlas Nacional de Cuba. La Habana: Instituto de Geografía de la Academia de Ciencias de Cuba e Instituto Cubano de Geodesia y Cartografía.

Lecha Estela, L. (1989). Humedad relativa media anual a las 7 y 13 horas. 1: 400000. Nuevo Atlas Nacional de Cuba. La Habana: Instituto de Geografía de la Academia de Ciencias de Cuba e Instituto Cubano de Geodesia y Cartografía.

León, H. (1946). Flora de Cuba 1. Gimnospermas. Monocotiledóneas. La Habana: Museo de Historia Natural Colegio La Salle.

León, H. \& Alain, H. (1951). Flora de Cuba 2. Dicotiledóneas: Casuarináceas a Meliáceas. La Habana: Museo de Historia Natural Colegio La Salle.

López-Portillo, J. \& Ezcurra, E. (1989a). Response of three mangroves to salinity in two geoforms. Functional Ecology, 3, 355-361. DOI: 10.2307/2389376

López-Portillo, J. \& Ezcurra, E. (1989b). Zonation in mangrove and salt marsh vegetation at Laguna de Mecoacán, México. Biotropica, 21, 107-114. DOI: $10.2307 / 2388701$

Magaz García, A.R. (1989). Ángulo de las pendientes. 11000000. Nuevo Atlas Nacional de Cuba. La Habana: Instituto de Geografía de la Academia de Ciencias de Cuba e Instituto Cubano de Geodesia y Cartografía.

McKee, K.L. (1993). Soil physicochemical patterns and mangrove species distribution-reciprocal effects? Journal of Ecology, 81, 477-487. DOI: $10.2307 / 2261526$

Martínez-Quesada, E. (2014). Nuevos sintáxones del archipiélago de los Jardines de la Reina, Cuba. Acta Bot. Malacitana, 39, 99-115.

Marrero Rodríguez, A., Pérez Jiménez, J., Suárez Estrada, E. \& Vega Lorenzo, E. (1989). Suelos. 1: 100000. Nuevo Atlas Nacional de Cuba. La Habana: Instituto de Geografía de la Academia de Ciencias de Cuba e Instituto Cubano de Geodesia y Cartografía.

Menéndez Carrera, L., Vilamajó, D. \& Herrera, P. (1987): Flora y vegetación de la cayería al norte de Matanzas, Cuba. Acta Botanica Cubana, 39, 1-20.

Menéndez Carrera, L. \& Priego Santander, A. (1994). Los manglares de Cuba: Ecología. In D. Suman (Ed.), El ecosistema de manglar en América Latina y la Cuenca del Caribe: su manejo y conservación (pp. 64-75). Miami, FL: Rosenstiel School of Marine and Atmospheric Science \& The Tinker Foundation.

Menéndez Carrera, L. \& Guzmán Menéndez, J.M. (2005). El humedal de Sabana-Camagüey: principales características de la vegetación. In J.J. Neiff (Ed.), Humedales de Iberoamérica: experiencia de estudios y gestión (pp. 146-153). Red Iberoamericana de Humedales (RIUH).

Menéndez Carrera, L. \& Guzmán Menéndez, J.M. (2007). Estado de los ecosistemas terrestres de los cayos. In P.M. Alcolado, E.E. García \& M. Arellano Acosta (Eds.), Ecosistema Sabana-Camagüey. Estado actual, avances y desafíos en la protección y uso sostenible de la biodiversidad (pp. 57-61). La Habana: Editorial Academia.
Menéndez Carrera, L., Guzmán Menéndez, J.M. \& Priego Santander, A. (2006). Manglares del Archipiélago Cubano: aspectos generales. In L. Menéndez Carrera \& J.M. Guzmán Menéndez (Eds.), Ecosistema de manglar en el Archipiélago Cubano (pp. 17-27). La Habana: Editorial Academia.

Nickerson, N.H. \& Thibodeau, F.R. (1985). Association between pore water sulfide concentratios and the distribution of mangroves. Biogeochemistry, 1, 183192. https://doi.org/10.1007/BF02185041

Pannier, F. \& Fraíno de Pannier, R. (1989). Los manglares de Venezuela. Caracas: Lagoven.

Peinado, M., Alcaraz, F., \& Delgadillo, J. (1995). Sintaxonomy of some halophilous communities of Nort and Central America. Phytocoenologia, 25(1); 23-31. DOI: $10.1127 /$ phyto/25/1995/23

Peinado, M., Alcaraz, F., Delgadillo, J., De la Cruz, M., Álvarez, J. \& Aguirre, J.L. (1994). The coastal salt marshes of California and Baja California. Phytosociological typology and zonation. Vegetatio, 110, 55-66. https://doi.org/10.1007/BF00038714

Peñalver Hernández, L.L. (1989). Constitución geológica. Depósitos cuaternarios.

1: 2000000. Nuevo Atlas Nacional de Cuba. La Habana: Instituto de Geografía de la Academia de Ciencias de Cuba e Instituto Cubano de Geodesia y Cartografía.

Portela Peraza, A.H., Díaz Díaz, J.L., Hernández Santana, J.R., Magaz García, A.R. \& Blanco Segundo, P. (1989). Geomorfología. Nuevo Atlas Nacional de Cuba. La Habana: Instituto de Geografía de la Academia de Ciencias de Cuba e Instituto Cubano de Geodesia y Cartografía.

Priego-Santander, A.G., Palacio Prieto, J.L., MorenoCasasola, P., López Portillo, J. \& Geissert Kientz, D. (2004). Heterogeneidad del paisaje y riqueza de flora: su relación en el archipiélago de Camagüey, Cuba. Interciencia, 29(3), 138-144.

Reyes Domínguez, O.J. (2005). Estudio sinecológico de las pluvisilvas submontanas sobre rocas del complejo metamórfico. Foresta Veracruzana, 7(2), 15-22.

Reyes Domínguez, O.J. (2006). Principales fitocenosis de manglares en el humedal del delta del río Cauto. In L. Menéndez Carrera, \& J.M. Guzmán Menéndez (Eds.), Ecosistema de manglar en el archipiélago cubano (pp. 263-270). La Habana: Editorial Academia.

Reyes Domínguez, O.J. \& Acosta Cantillo, F. (2003). Fitocenosis presentes en las áreas costeras del sur de la Sierra Maestra, Cuba. I. Comunidades con influencia marina. Foresta Veracruzana, 5(2), 1-7.

Ricardo, N.E., Herrera, P. \& Pouyú, E. (1990). Clasificación de la flora sinantrópica de Cuba. Revista del Jardín Botánico Nacional, Universidad de La Habana, 11(2), 129-133.

Ricardo, N.E., Pouyú, E. \& Herrera, P. (1995). The synanthropic flora of Cuba. Fontqueria, 42, 367-430.

Romero-Jiménez, M., Castañeda-Noa, I. \& MásCastellanos, L.M. (2015). Conservación y sinantropismo en áreas naturales de Cayo Las Brujas, Villa Clara, Cuba. Revista del Jardín Botánico Nacional, Universidad de La Habana, 36, 79-91.

Samek, V. (1973). Pinares de la Sierra de Nipe. Estudio Sinecológico. Academia de Ciencias de Cuba, serie 
Forestal, 14, 1-58.

Scamoni, A. \& Passarge, H. (1963). Einführung in die praktische Vegetationskunde. 2. Jena: G. Fischer.

Trejo, I. \& Dirzo, R. (2002). Floristic diversity of Mexican seasonally dry tropical forest. Biodiversity and Conservation, 11, 2063-2084.

Tolinson, P.B. (1986). The botany of mangroves. Cambridge: Cambridge University Press.

Valdés Montero, J.F. (2000). Diccionario geográfico de Cuba. Comisión Nacional de Nombres Geográficos. Oficina Nacional de Geografía y Geodesia. La
Habana: Ediciones GEO.

Vilamajó, D. \& Vales, M.A. (2006). Aspectos de la ecoanatomía y ecomorfología foliar de los manglares cubanos. In L. Menéndez Carrera \& J.M. Guzmán Menéndez (Eds.), Ecosistema de manglar en el Archipiélago Cubano (pp. 17-27). La Habana: Editorial Academia.

Weber, J.E., Moravec, J. \& Theurillat, J.P. (2000). International Code of Phytosociological Nomenclature. 3rd edition. Journal of Vegetation Science, 11, 739768. DOI: $10.2307 / 3236580$ 
